\title{
2 Von der Bewegung zur NGO (ca. 1968-1973)
}

\subsection{8: Die Barthianer fordern von der Schweiz die Umsetzung der UNCTAD-Forderungen}

Im Vergleich zu anderen europäischen Ländern verlief das Jahr 1968 in der Schweiz harmlos. ${ }^{1}$ Hier waren die Studierenden nicht mit der Rolle der Elterngeneration im Holocaust konfrontiert wie in Deutschland, aber auch nicht mit der schwarzen Bürgerrechtsbewegung oder dem Vietnamkrieg wie in den USA. Auch bildete sich in der Schweiz nicht wie in Grossbritannien oder den Niederlanden eine Bürgerbewegung als Antwort auf die Aktivitäten in den eigenen Kolonien. ${ }^{2} \mathrm{Zu}$ Recht schreibt Bernhard C. Schär: „Für die Erforschung und Deutung von 1968 in der Schweiz dürfte es daher ertragreich und spannend sein, neben den Brüchen auch die Kontinuitäten ins Auge zu fassen.." ${ }^{\text {3 }}$ Diese Kontinuität sticht bei den Verfassern der „Erklärung von Bern“ ins Auge. Es handelte sich bei ihnen um eine durchaus arrivierte, ältere und bürgerliche Gruppe von Theologen. Interessant ist allerdings auch hier, wie Slobodian aufgezeigt hat, der Dialog mit dem Süden, für den sich nicht nur junge Studenten, sondern auch die Vätergeneration einsetzte. ${ }^{4}$ Ganz im Sinne der UNCTAD, dem neuen Zusammenschluss der Entwicklungsländer, setzten sich die Initianten der „Erklärung von Bern“ für Wachstum, Modernisierung und Industrialisierung ein. Sie forderten das Aufschliessen der Entwicklungsländer $\mathrm{zu}$ den Industrieländern. Interessant ist auch, wie hoffnungsvoll die Vertreter der Vätergeneration in die Zukunft blickten und den Umbruch willkommen hiessen. ${ }^{5}$

Die Nähe der Gruppe der Theologen zum Bürgertum zeigt sich auch an deren Haltung zur Schweizer Wirtschaft. Gemäss der „Neuen Zürcher Zeitung“ (NZZ) forderten die Initianten auf der Pressekonferenz zur „Erklärung von Bern“ im Januar 1969, dass auch die Schweizer Industrie einen Beitrag zur Entwicklung der armen Länder leiste. ${ }^{6}$ Dies war ein Modernisierungs- bzw. Industrialisierungsplädoyer. Repetiert wurde gemäss der NZZ der UNCTAD-Vorwurf, die Schweizer Industrie investiere nicht in die Entwicklungsländer bzw. modernisiere dort nicht, sie schöpfe nur den billigen Rohstoff ab. Das heisst, 1968 erwarteten die Initianten

1 B. C. Schär: ,1968‘ als wiederbelebte bürgerliche Revolution, 7.

2 R. Gildea, J. Mark, A. Warring: Europe’s 1968.

3 B. C. Schär, R. Ammann, T. Färber, S. Bittner, M. Hofer, Y. Niederhäuser, V. Sperisen, M. Griesshammer, R. Fischer, M. Schär, R. Schär, E. Vaudan: Bern 68, 7.

4 Q. Slobodian: Foreign Front, 18-26.

5 J. Rossel: Dynamik der Hoffnung, 14.

6 Ohne Autor, Ein Appell zur Entwicklungshilfe, NZZ, 10.1.1969.

Ә OpenAccess. (c) 2020 Andrea Franc, publiziert von De Gruyter. (cc) BY-NC-ND Dieses Werk ist lizenziert unter der Creative Commons Attribution-NonCommercial-NoDerivatives 4.0.

https://doi.org/10.1515/9783110667516-006 
von den Schweizer Firmen geradezu, dass sie in Entwicklungsländern Fabriken bauen und die Rohstoffe vor Ort verarbeiten sollten. Von Schweizer Firmen wurde erwartet, dass sie ihren Beitrag zur Industrialisierung der Dritten Welt leisteten. Die Privatwirtschaft wurde in der „Erklärung von Bern“ nicht explizit, sondern nur mündlich anlässlich der Pressekonferenz erwähnt. Peter Gesslers schriftliche Notizen zur Pressekonferenz sind jedoch unmissverständlich:

Sie [die Erklärung von Bern] fordert die Bundesversammlung auf,

1. eine Zoll- und Handelspolitik unseres Landes zu ermöglichen, die eine gerechtere Verteilung der Reichtümer der Erde zum Ziel hat und den wirtschaftlichen Aufstieg der Entwicklungsländer fördert. Die Sonderinteressen der Schweizerischen Wirtschaft müssen dabei unter Umständen zurücktreten.

2. den Empfehlungen der Welthandelskonferenz von Neu-Delhi Folge zu leisten und Zollund Einfuhrbarrieren besonders bei Industrieprodukten aus Entwicklungsländern fallen zu lassen.

3. entsprechend den Empfehlungen der Welthandelskonferenz von Neu-Delhi und der Weltkirchenkonferenz von Uppsala sich dafür einzusetzen, dass bis 1971 aus öffentlichen Mitteln mindestens 1 Prozent des Bruttosozialproduktes der Schweiz zusätzlich zu den Investitionen der Privatindustrie in geeigneter Form für die Entwicklungsländer eingesetzt wird. ${ }^{7}$

Die Barthianer hatten es sich zum „Auftrag“ gemacht, die „Empfehlungen der Welthandelskonferenz von Neu-Delhi“, der zweiten UNCTAD von 1968, in der Schweiz zu vertreten. ${ }^{8}$ Die Schweiz sollte gemäss Manifest „auf bestimmte Privilegien verzichten“, nämlich „Zoll- und Einfuhrbarrieren besonders bei Industrieprodukten aus Entwicklungsländern“ abschaffen und in Kauf nehmen, dass „Sonderinteressen der Schweizer Wirtschaft“ geschädigt werden. ${ }^{9}$ Klar sahen die Initianten „Investitionen der Privatindustrie“ als Hilfe zum „wirtschaftlichen Aufstieg der Entwicklungsländer“, diese mussten jedoch durch einen solidarischen Finanzausgleich ähnlich dem der Schweizer Kantone ergänzt werden, um der Verschlechterung der realen Austauschverhältnisse entgegenzuwirken. ${ }^{10}$

7 SozArch, EvB, Ar 430.25.1. EvB, Anfangszeit: Die Erklärung von Bern: Die Schweiz und die Entwicklungsländer. Peter Gessler, 10.1.1969.

8 Zu diesem „Auftrag“ siehe SozArch, EvB, Ar 430.25.1. EvB, Anfangszeit: André Biéler, Max Geiger, Lukas Vischer an „Freunde und Brüder“, „Anfang“ Dezember 1967.

9 Siehe Punkt 5 des Manifests. SozArch, EvB, Ar 430.25.1. EvB, Anfangszeit: Die Erklärung von Bern, März 1968.

10 SozArch, EvB, Ar 430.25.1. EvB, Anfangszeit: Die Beziehungen der Schweiz zu den Entwicklungsländern, Entwurf M. Geiger (Punkt 5 Dr. Peter Gessler), undatiert. 
Hauptsächlich forderten die Initianten im Manifest vom Schweizer Staat die handelspolitische Umsetzung der UNCTAD-Forderungen. Dies bedeutete die Forderung nach Marktzugang für die Entwicklungsländer, die Einführung von Zollpräferenzen, die Unterstützung der Modernisierung und deren import substitution investment. ${ }^{11}$ Nachlesen lässt sich diese Haltung in einem ersten, ausführlichen Entwurf der Erklärung, in dem Max Geiger schreibt, dass die Industrialisierung der Entwicklungsländer nicht erschwert werden dürfe. ${ }^{12}$ Interessant im Hinblick auf die kommende Wandlung der Idee des gerechten Handels ist zudem, dass in diesem Entwurf Geigers und Gesslers die Landwirtschaft kurz erwähnt wird, danach aber zugunsten des Themas Handelspolitik und Wirtschaftswachstums gestrichen wurde. In dem ersten ausführlichen Entwurf fordert Gessler nicht nur, dass Entwicklungsländer eigene Industrien aufbauen und auf dem Weltmarkt als Konkurrenten auftreten können, sondern auch einen Strukturwandel der Schweizer Landwirtschaft:

Der Agrarprotektionismus der Industrieländer führt immer wieder zu Zusammenbrüchen der Weltmarktpreise derjenigen agrarischen Produkte in Entwicklungsländer, die in Konkurrenz zu Produkten der entwickelten Länder stehen (Fettstoffe, Zucker, Textilfasern u.a.). Die Schweiz muss dazu beitragen, dass die Agrarwirtschaft vom kleinräumigen Denken auf eine weltwirtschaftliche Basis umgestellt wird. ${ }^{13}$

Wäre die „Erklärung von Bern“ länger gewesen und hätte diese Passage Eingang gefunden, so wäre der Konzeptwandel in den folgenden Jahren augenfälliger gewesen. Klar wird hier der „Agrarprotektionismus der Industrieländer“ (und nicht wie später die Agrarkonzerne) für die starke Preisvolatilität der Agrarrohstoffe benannt. Ebenso klar wird das „kleinräumige Denken“ noch verurteilt, während die EvB ab Mitte der 1970er-Jahre die ,internationale Arbeitsteilung“ in der Landwirtschaft verurteilte. ${ }^{14}$ Noch sahen die Initianten jedoch die Schweiz als Industrienation, ihren Industrie- und Agrarprotektionismus sowie insbesondere ihre multinationalen Unternehmen und deren „Welthandelstradition“ in der Pflicht. ${ }^{15}$

11 Siehe dazu S. Misteli: Der UNCTAD-Moment sowie T. Brodbeck: Bewahren und beharren. 12 SozArch, EvB, Ar 430.25.1. EvB, Anfangszeit: Die Beziehungen der Schweiz zu den Entwicklungsländern, Entwurf M. Geiger (Punkt 5 Dr. Peter Gessler), undatiert.

13 SozArch, EvB, Ar 430.25.1. EvB, Anfangszeit: Die Beziehungen der Schweiz zu den Entwicklungsländern, Entwurf M. Geiger (Punkt 5 Dr. Peter Gessler), undatiert.

14 Sieh dazu etwa A.-M. Holenstein, J. Davis: Zerstörung durch Überfluss, 58.

15 SozArch, EvB, Ar 430.25.1. EvB, Anfangszeit: Die Beziehungen der Schweiz zu den Entwicklungsländern, Entwurf M. Geiger (Punkt 5 Dr. Peter Gessler), undatiert. 
Im November 1970 wiederholte die EvB in einem schriftlichen, veröffentlichten Positionspapier diese Haltung und setzte eine Passage der Erklärung nochmals präziser in den Kontext der UNCTAD-Forderungen:

Unsere überseeischen Investitionen: nicht Alibi, sondern Aufgabe

An ihnen ist es, die Infrastrukturen der Entwicklungspartner aufbauen zu helfen und deren Wachstum zu politischer, ökonomischer und kultureller Selbständigkeit zu fördern („Erklärung von Bern“, art. 4). ${ }^{16}$

Dieser ursprüngliche Einsatz für private Direktinvestitionen im Süden ging aber bereits in den darauf folgenden Jahren verloren. Die Theologen der Anfangszeit der EvB hatten in erster Linie in ihrer Funktion als Pfarrer in der Gesellschaft agiert. Ihr Engagement für die Erklärung war unbezahlt, ehrenamtlich und dadurch auch entsprechend spontan. Die Medien nahmen vor allem ihren Aufruf, drei Prozent des eigenen Vermögens an die Entwicklungshilfe zu spenden, auf und stilisierten dies zum Hauptpunkt der Erklärung. ${ }^{17}$

Die Menschengruppe hinter der Erklärung blieb weiterhin eine lose Gemeinschaft, die sich im Rahmen von kirchlichen Veranstaltungen weiterhin traf. Im November 1970 organisierten Vertreter der Kirche die interkonfessionelle Konferenz Schweiz - Dritte Welt, die im Bundeshaus bzw. im Nationalratssaal abgehalten werden durfte. Kalt hat diese Konferenz in ihrer Dissertation ausführlich beschrieben und beleuchtet. Sie hat aufgezeigt, wie sich damals erstmals eine „Jugendfraktion“ um den jungen Ökonomen Rudolf Strahm und den katholischen Priester Al Imfeld bildete. ${ }^{18}$ Die „Jugendfraktion“ setzte sich von den Barthianern ab und formulierte eigene Anliegen. Somit bildete sich erst zwei Jahre nach 1968 eine aktive Jugendbewegung im Umfeld der „Erklärung von Bern“, die sich durchaus gesittet gegen die Vätergeneration auflehnte. Sie tat dies innerhalb eines bereits seit längerem von der Vätergeneration in Gang gesetzten Prozesses, in dem die Frage nach der Nord-Süd-Gerechtigkeit thematisiert wurde. Der Hauptpunkt, in dem die Jungendfraktion die Vätergeneration kritisierte, war die Frage, ob die Industrialisierung der Entwicklungsländer durch private Direktinvestitionen westlicher Firmen oder staatliche Investitionen der Entwicklungsländer selbst erfolgen sollte. Es gelang der Jugendfraktion, ihre kritische Haltung gegenüber den Auslandinvestitionen der Schweizer Privatwirtschaft bereits in den

16 SozArch, EvB, Ar 430.25.1, EvB, Anfangszeit, EvB: Die Schweiz und die Entwicklungsländer, Text, der am 14.11.1970 in Bern vom Schweizer Komitee der EvB genehmigt wurde.

17 A.-M. Holenstein-Hasler, R. Renschler, R. H. Strahm: Entwicklung heisst Befreiung, 25.

18 M. Kalt: Tiersmondismus in der Schweiz, 296-303. 
Konferenzbericht einfliessen zu lassen. ${ }^{19}$ Mit dem Rückzug der Vätergeneration aus dem Aufbau der Organisation EvB setzte sich die Haltung der Jugendfraktion definitiv durch. ${ }^{20}$

Die interkonfessionelle Konferenz vom November 1970 war zudem der erste Schritt in Richtung einer organisierten Institutionalisierung der Bewegung. Auch dieser Prozess wurde von den etablierten Kirchen ausgelöst und zunächst auch verfolgt. Die Jungen konnten sozusagen auf den Zug aufspringen und sich als Sekretär der EvB (Strahm) oder Geschäftsführer des von der Arbeitsgemeinschaft der Hilfswerke geschaffenen Informationsdienstes Dritte Welt (Imfeld) anstellen lassen. ${ }^{21}$ Dieser Prozess der Institutionalisierung und der Beginn des Organisationswachstums - wie er auch bei Hilton als typischer Prozess in der Entwicklung von britischen NGOs in den 1970er-Jahren beobachtet wird - kam nach der interkonfessionellen Konferenz ins Rollen. ${ }^{22}$ Die Zeit zwischen 1968 und 1970 war jedoch noch eine Phase, in der die Barthianer Max Geiger, Lukas Vischer oder Kurt Marti, der Calvinist André Biéler oder der Missionar Jacques Rossel in ihrer Autorität als Professoren oder Pfarrer ehrenamtlich das Anliegen der Nord-Süd-Gerechtigkeit vertraten.

Die Konferenz von 1970 beflügelte das Organisationswachstum und war auch ein erster subtiler Schritt hin zu einem Wandel des Fair-Trade-Konzepts innerhalb der Bewegung. Dieser Schritt dürfte denn auch auf den Einfluss der von Kalt beschriebenen „Jugendfraktion“ zurückzuführen sein, die sich nach 1968 aktuelle Schriften der kapitalismuskritischen Dependenztheorie, darunter Johan Galtung, Rodolfo Stavenhagen oder Dieter Senghaas, zu Gemüte geführt hatte und diese nun gegen die Barthianer ins Feld führte. ${ }^{23}$ Auch Regula Renschler, die 1974 zur Erklärung von Bern stiess, nahm als Redaktorin der „Schweizer Arbeiterzeitung“ an der Konferenz teil. ${ }^{24}$ Zudem lernten sich Christoph Eckenstein und Roy Preiswerk bei den Vorbereitungen zur Konferenz kennen. Die beiden Männer hatten vieles gemeinsam, sie stammten aus Basler Bürgerfamilien, hatten in Genf Recht studiert und für den Bund, aber auch für Entwicklungsländer gearbeitet. Sie setzten sich etwas von der Gruppe bürgerlicher Schweizer Theologen um die „Erklärung von Bern“ ab. Von diesem Zeitpunkt an verband die beiden eine tiefe

19 M. Schär: Strukturveränderungen statt Entwicklungshilfe, 84-85.

20 Siehe dazu Kapitel 3.3. Der implizite Konsens zur Ablehnung von Privatinvestitionen in Entwicklungsländern.

21 M. Kalt: Tiersmondismus in der Schweiz, 296.

22 M. Hilton: A Historical Guide, 347-350.

23 Siehe dazu etwa A.-M. Holenstein-Hasler, R. Renschler, R. H. Strahm: Entwicklung heisst Befreiung, 151 oder M. Schär: Strukturveränderungen statt Entwicklungshilfe, 76.

24 A.-M. Holenstein-Hasler, R. Renschler, R. H. Strahm: Entwicklung heisst Befreiung, 97. 
Freundschaft und gemeinsam setzten sie sich für die „Jugendfraktion“ ein und traten mit Rudolf Strahm und Al Imfeld in einen Dialog..$^{25}$

Diese personellen Veränderungen und Anpassungen spiegeln auch die ersten konzeptuellen Änderungen wider. Wie aufgezeigt, stimmten die Initianten der „Erklärung von Bern“ zunächst durchaus mit der Schweizer Privatwirtschaft überein und machten dieser im Gegenteil Vorwürfe, weil sie nicht mehr in Entwicklungsländer investierte. Hier ging die „Jugendfraktion“ weiter als die UNCTAD-Forderungen und kritisierte die westlichen Grosskonzerne grundsätzlich. Vor allem Roy Preiswerk vermochte den Jungen hier doch bis zu einem gewissen Grad zu folgen, beschäftigte er sich doch bereits zu dieser Zeit mit den kulturellen Folgen der westlichen privaten Direktinvestitionen. Insbesondere aber waren Preiswerk und Eckenstein mit den Bestrebungen der Entwicklungsländer vertraut, eine eigene Industrie aufzubauen. Der Schwerpunkt verschob sich somit in Richtung einer generellen Ablehnung westlicher privaten Direktinvestitionen. Ebenfalls wird bereits 1970 unterschwellig die Suche nach alternativen Formen der Produktion in Entwicklungsländern erkennbar. Diese Alternativen wurden von der jüngeren Generation zunächst noch etwas plump als sozialistische Alternative gepriesen, während Preiswerk die Frage nach der kulturellen Vereinbarkeit von Privatinvestitionen in Ländern des Südens stellte.

\subsection{0: Eine Dritte-Welt-Konferenz im Bundeshaus: erste Anpassungen}

Zwei Jahre nach dem Treffen der Initianten der „Erklärung von Bern“, das 1968 in Gwatt stattfand, kam es im November 1970 zu einem zweiten, noch grösseren Treffen. Es handelte sich wiederum um eine kirchliche Zusammenkunft, doch diese fand nicht in einem kirchlich geprägten Umfeld, sondern im Bundeshaus statt, und die jungen und die säkularen Vertreterinnen und Vertreter mit ihren Wirtschaftsthemen - beispielsweise Christoph Eckenstein und Roy Preiswerk waren dabei. Allein der Ort der Tagung, das Bundeshaus, symbolisierte, dass die Bewegung von 1968 den gar nicht so langen Marsch durch die Institutionen angetreten hatte, und besiegelte die Stabsübergabe von der Kirche an noch zu entstehende, säkulare Organisationen. Die schriftliche Ausbeute der Konferenz war diesmal ausführlicher: Das 13-Punkte-Manifest von 1968 wurde zu einem über 100

25 R. Preiswerk, G. Rist: A contre-courants, 50, 54. 
Seiten umfassenden Büchlein ausgebaut. ${ }^{26}$ Darin war die persönliche christliche Handlung, die im Manifest unter der Spende von drei Prozent festgelegt war, nicht mehr erwähnt. Dafür erhielt die Wirtschafts- und Handelspolitik ein umso stärkeres Gewicht und wurde zum Hauptthema. ${ }^{27}$ Die Nord-Süd-Frage war nun gänzlich zur Frage nach einem gerechten Weltwirtschaftssystem und der Rolle, welche die Schweiz darin spielen sollte, geworden. So stellte die Konferenz unter dem Titel „Einige grundlegende Tatsachen“ Folgendes fest:

Jedes Land ist auf den Warenaustausch mit dem Ausland angewiesen. Die armen Länder sind besonders stark von den Exporterlösen abhängig, die ihnen dazu dienen, die für ihr wirtschaftliches Wachstum notwendigen Importe aus den industrialisierten Ländern zu bezahlen. $^{28}$

Damit beugten sich auch die Theologen auf der Konferenz von 1970 dem Primat der Wirtschaft. Ein Prozess, der mit André Biélers Doktorarbeit in Wirtschaftswissenschaften in den 1950er-Jahren symbolisch begonnen hatte, fand hier seinen Kulminationspunkt. Doch die Konferenz von 1970 war nur ein Blitzlicht in der Entwicklung der Fair-Trade-Bewegung oder eine Art kurzes ökonomisches Fenster, das sich bald wieder schliessen würde. Gleich mehrere konzeptuelle Aspekte, die später ins Gegenteil verkehrt werden sollten, bilden hier die Grundlage. Da ist zunächst die Beobachtung, dass jedes Land auf den Warenaustausch angewiesen ist. Gerade dieses Primat des Handels und die positive Sicht des Handels werden später implizit wiederrufen. Nicht nur für Entwicklungsländer wurde die Selbstversorgung, die Abkopplung und das „Weniger nehmen“ für die Industrieländer propagiert, auch für die Schweiz bzw. für die Industrieländer wurde die lokale, und damit faktisch die nationale Produktion Mitte der 1970er-Jahre wieder zum Ziel. $^{29}$ Der Erhalt Schweizer Arbeitsplätze, die lokale landwirtschaftliche Produktion und die „Bewahrung“ der Entwicklungsländer vor Ausbeutung und Umweltverschmutzung sollten diese „Grundlagen“, wie sie 1970 noch genannt

26 Schmocker, Hans; Traber, Michael (Hg.): Schweiz-Dritte Welt. Berichte und Dokumente der Interkonfessionellen Konferenz in Bern (Zürich, Freiburg 1971).

$27 \mathrm{Zu}$ dieser kurzen Phase des Primats der Handelspolitik siehe auch Saunders, Clare: British Humanitarian, Aid and Development NGOs, 1949-Present, in: N. J. Crowson, Matthew Hilton, James McKay (Hg.): NGOs in Contemporary Britain. Non-State Actors in Society and Politics since 1945 (Basingstoke 2009) 38-58.

28 H. Schmocker, M. Traber: Schweiz-Dritte Welt, 103.

29 Siehe dazu Kapitel 4.2. Der Kleinbauer als gemeinsamer politischer Nenner: ein Bio-Gütesiegel 1981, die Futtermittelinitiative und die Kleinbauerninitiative. Siehe auch K. J. Kuhn: Entwicklungspolitische Solidarität, 264. 
wurden, ausser Kraft setzen und langfristig die Autarkie zum Idealfall stilisieren. ${ }^{30}$

Auffällig ist im obigen Zitat auch die Formulierung, wonach für die armen Länder „wirtschaftliches Wachstum notwendig“ sei. Wie stark sollten sich die Akzente schon wenige Jahre später verschieben! Die Theologen und jugendlichen Aktivisten der Konferenz sahen das wirtschaftliche Wachstum der Entwicklungsländer als Ziel und erachteten dies als positiv. Dass die Entwicklungsländer dafür der Falle der Produktion von Agrarrohstoffen entkommen und verarbeitende Industrie aufbauen mussten, bezeichnete die Konferenz als „grundlegende Tatsache“. Damit deckte sich die Haltung der Schweizer Dritte-Welt-Bewegung mit der zeitgenössischen wirtschaftswissenschaftlichen Forschung sowie den Forderungen der Entwicklungsländer. ${ }^{31}$ Dies zeigt sich auch in Punkt 4 der ,grundlegenden Tatsachen“:

Die Entwicklungsländer weisen darauf hin, dass es nicht nur darum geht, ihre Bedingungen des Warenaustausches zu stabilisieren, sondern diese zu verbessern mit dem Ziel, die Kluft zwischen armen und reichen Nationen kleiner werden zu lassen. ${ }^{32}$

Hier sind wiederum zwei Aspekte interessant. Zum einen wieder die utopische oder zumindest dynamisch-zukunftsgerichtete Aussage, dass die Kluft zwischen den Nationen kleiner werden soll. Bis zu Beginn der 1970er-Jahre gingen die Aktivisten also noch von einem einheitlichen Konzept von Entwicklung oder Zivilisation aus, das für alle Völker der Erde galt und in einem Bruttosozialprodukt gemessen werden konnte. ${ }^{33}$ Der Unterschied zwischen den Werten dieses Bruttosozialprodukts sollte kleiner werden. Eine Vorstellung von unterschiedlichen Arten der Entwicklung, die in einem Bruttosozialprodukt nicht zum Tragen kam, schien noch nicht diskutiert worden zu sein. Es gab nur eine Messart für arm und reich, die in den Wirtschaftswissenschaften wiederum weiterbestehen sollte, wenn auch in einer sehr verfeinerten Indexierung. Weder Vorläufer der Wachstumskritik noch des Slogans Small is beautiful, der die Armut aufwertete, hatten sich in diesen Text verirrt. Er zeigt, welch grundlegend anderes und auf eine

30 Siehe dazu Kapitel 4.5 Für Protektionismus und Autarkie, gegen Freihandel und Grosskonzerne: das Fair-Trade-Konzept der Erklärung von Bern zu Beginn der 1980er-Jahre. Siehe auch M. Schär: Strukturveränderungen statt Entwicklungshilfe, 38.

31 J. Toye, R. Toye: UN and Global Political Economy, 147.

32 H. Schmocker, M. Traber: Schweiz-Dritte Welt, 104.

33 SozArch, EvB, Ar 430.25.1. EvB, Anfangszeit: Einige Zahlen zur Orientierung, undatiert, ohne Autor (als Beilage der „Erklärung von Bern“ zur Information für Unterzeichner). Siehe dazu auch Speich Chassé, Daniel: Die Erfindung des Bruttosozialprodukts. Globale Ungleichheit in der Wissensgeschichte der Ökonomie (Göttingen 2013) 210. 
wirtschaftswissenschaftliche Sichtweise basierendes Konzept die schweizerische Dritte-Welt-Bewegung 1971 noch vertrat.

Zweitens wird in diesem Textzitat wiederum deutlich, dass die anfängliche Bewegung eine Reaktion war. Mit der Formulierung „Die Entwicklungsländer weisen darauf hin“ kann nur die UNCTAD gemeint sein, die damals der prominenteste Zusammenschluss von Entwicklungsländern mit geeinter Stimme war. ${ }^{34}$ „Die Entwicklungsländer“ werden als aktive und fordernde, als ernst zu nehmende Gruppe betrachtet. Es wurde davon ausgegangen, dass sie ihren Rückstand in Zukunft aufholen würden. ${ }^{35}$ Eine „solidarische Entwicklung“, wie es die EvB in ihren Statuten von 1970 nannte, basierte auf dieser Sichtweise. ${ }^{36}$ Eine „solidarische Entwicklung“ bedeutete, die Entwicklungsländer in ihrer Entwicklung zu Industrieländern zu unterstützen. Dafür brauchte es, wie es in der „Erklärung von Bern“ von 1968 bereits implizit steht, den Marktzugang zu Industrieländern, den Aufbau einer eigenen Industrie und - dies wird hier im Text von 1971 noch genauer ausgeführt - die regionale Integration, das heisst etwa den Freihandel unter benachbarten afrikanischen, asiatischen oder lateinamerikanischen Staaten. Dies war Christophe Eckensteins Kernanliegen. Bereits 1967 hatte die UNO einen Bericht Eckensteins über die regionale Integration unter Entwicklungsländern publiziert. ${ }^{37}$ Die Konferenz setzte sich also wortwörtlich für eine regionale Handelsliberalisierung im Süden ein. Dass der Begriff „Integration" verwendet wurde, der wirtschaftswissenschaftliche Fachbegriff für Handelsbeziehungen, zeugt vom Fachwissen der Autoren. So forderte die Konferenz von der Schweiz eine Entwicklungspolitik, die sich systematisch für die regionale Integration im Süden einsetzte: „Eine solche Politik systematischer Unterstützung von Handelsliberalisierungs- und regionalen Integrationsbemühungen soll in möglichst konkreter Weise definiert werden. “38 Gerade die Tatsache, dass sich die Schweizer Konferenz so ausdrücklich für regionale Handelsliberalisierung einsetzte, ist im Lichte der aktuellen Forschung, die Entwicklungsorganisationen als Gegner der regionalen Integration benennt, besonders interessant. ${ }^{39}$ Daher ist es wichtig, darauf hinzuweisen, dass sich die Dritte-Welt-Bewegung im Jahr 1971

34 J. Toye, R. Toye: UN and Global Political Economy, 233.

35 M. Schär: Strukturveränderungen statt Entwicklungshilfe, 5.

36 Siehe SozArch, EvB, Ar 430.10.1. Mappe 2: Entwurf für die Statuten der EvB.

SozArch, EvB, Ar 430.11.1. Mappe 3: Statuten der schweizerischen Vereinigung zur Föderung der EvB für solidarische Entwicklung.

SoZArch, EvB, Ar 430.10.2. Mappe 3: Neue Statuten 1977: div. Statutenrevisionen.

37 C. Eckenstein, B. Gurtner: Den Dialog erkämpfen, 14.

38 H. Schmocker, M. Traber: Schweiz-Dritte Welt, 115.

39 P. Collier: The Bottom Billion, 157-159. 
noch anders präsentiert hatte und sich an die wirtschaftswissenschaftlichen Grundlagen gehalten hatte.

Die Konferenz stellte sich klar gegen wichtige inländische Anliegen. Im Gegensatz zum Manifest von 1968 wird im Text von 1971 auch der Teilbereich Landwirtschaft separat angesprochen und der Agrarprotektionismus, der sich 1971 erst im Anfangsstadium befand, wird aus entwicklungspolitischer Sicht klar abgelehnt:

Mit Bezug auf die landwirtschaftlichen Produkte, die sowohl in Entwicklungsländern als auch in der entwickelten Welt produziert werden, anerkennen wir, dass die Entwicklungsländer ihren gegenwärtigen Marktanteil bei uns halten können sollten, wenigsten bei all denjenigen Produkten, wo sie ohne Subventionen konkurrenzfähig sind. Auf jeden Fall sollten wirksame Massnahmen getroffen werden, damit nicht-subventionierte Importe aus Entwicklungsländern auf unserem Markt nicht durch Importe subventionierter Produkte aus entwickelten Ländern verdrängt werden können; in dieser Hinsicht werden die Bundesbehörden auch ersucht zu prüfen, inwiefern eine solche Reduktion des Marktanteils der Einfuhren aus Entwicklungsländern in der Vergangenheit schon erfolgt ist. Die Schweiz sollte überdies keine handelspolitischen Abmachungen mit anderen Industrieländern treffen, welche die Absatzbedingungen in der Schweiz von nicht-subventionierten Produkten aus Entwicklungsländern erschweren..$^{40}$

Durch dieses Quellenzitat gelangt man wiederum zu erstaunlichen Einsichten. Ein Bericht über die „Reduktion des Marktanteils der Einfuhren aus Entwicklungsländern in der Vergangenheit“ bei Agrarrohstoffen scheint nie vorgelegt worden zu sein. Und wenn ein solcher Bericht Jahre später erstellt worden sein sollte, dann wurde er von der Dritte-Welt-Bewegung nicht mehr rezipiert. Das Thema Marktanteil der Agrareinfuhren aus Entwicklungsländern fiel nach der Konferenz unter den Tisch bzw. wurde durch die Wachstumskritik und das Aufkommen des biologischen Landbaus im Inland komplett verdrängt. Allein die „grundlegende Tatsache“, dass es „landwirtschaftliche Produkte“ gibt, „die sowohl in Entwicklungsländern wie in der entwickelten Welt produziert werden“, war aus der Wahrnehmung der Dritte-Welt-Bewegung verschwunden. ${ }^{41}$ Hochinteressant ebenfalls, wie hier die frühe Dritte-Welt-Bewegung im Bundeshaus aus entwicklungspolitischer Sicht Vorbehalte gegen einen Beitritt der Schweiz zu den europäischen Vertragswerken anbrachte. Denn bei der Gemeinsamen Europäischen Agrarpolitik (GAP) der EWG ging es genau um den Sachverhalt, den die Konferenz beschrieb, nämlich um „handelspolitische Abmachungen mit anderen Industrieländern, welche die Absatzbedingungen in der Schweiz von nicht-sub-

40 H. Schmocker, M. Traber: Schweiz-Dritte Welt, 108-109.

41 Dieser Aspekt wird in den untersuchten Quellen nicht mehr erwähnt. 
ventionierten Produkten aus Entwicklungsländern erschweren“.42 Hier wurde denn auch die Europakritik der Dritte-Welt-Bewegung sichtbar. Mit der ebenfalls 1971 erschienenen Studie mit dem Titel „EWG und Dritte Welt“ wurden die Initianten der „Erklärung von Bern“ noch sehr viel konkreter mit ihrer Europakritik. ${ }^{43}$ Diese Europa-Skepsis blieb im Fair-Trade-Konzept erhalten, zumal sie auch ein Aspekt war, der mit dem breiten Schweizer Konsens, dem gemeinsamen politischen Nenner, zu vereinbaren war. ${ }^{44}$ So hätte sich die EvB die Auftragsstudie sparen können, welche die entwicklungspolitischen Aspekte eines allfälligen Beitritts der Schweiz zur EWG durchspielte. In keiner Weise hatte die Mehrheit der Schweizer Bürgerlichen vor, der EWG beizutreten.

1970, im selben Jahr, in dem die Konferenz im Bundeshaus stattfand, wurde der Berner Ökonom Gerhard Winterberger (1922-1993) als Direktor des Schweizerischen Handels- und Industrieverbandes, des Vororts, eingesetzt, nachdem er bereits seit 1961 als volkswirtschaftlicher Sekretär die Richtung des Verbandes geprägt hatte. ${ }^{45}$ Winterberger war seit 1959 Mitglied der neoliberalen Mont Pèlerin Society und pflegte mit zahlreichen neoliberalen Ökonomen freundschaftlichen Kontakt. ${ }^{46}$ Winterberger repräsentierte somit nicht nur die schweizerische Privatwirtschaft und die Interessen der bürgerlichen Schweiz, sondern auch die Geisteshaltung neoliberaler Denker im weiteren Sinne. Bereits 1961 hielt er sowohl die seiner Ansicht nach politischen wie auch die wirtschaftlichen Gründe für einen Nicht-Beitritt der Schweiz zur EWG fest:

Es war von Anfang an klar erkennbar, dass die Schweiz aus politischen und wirtschaftlichen Gründen der Europäischen Wirtschaftsgemeinschaft als Mitglied nicht beitreten kann. Schweizerische Eigenart und Wesensmerkmale der Europäischen Wirtschaftsgemeinschaft stehen einander diametral gegenüber. [...] Es ist vollständig klar, dass eine Abtretung der Souveränität auf diesen Gebieten an supranationale Behörden dem schweizerischen Nationalgedanken und ihren Trägern, dem föderalistischen Staatsaufbau, der Gemeindeautonomie und der direkten Demokratie, den Todesstoss versetzen und unsere politische Lebensform auslöschen würde. ${ }^{47}$

42 M. Spoerer: Fortress Europe.

43 Wagner, Antonin: EWG und Dritte Welt. Die entwicklungspolitischen Implikationen eines EWG-Beitritts der Schweiz (Zürich 1971).

44 Siehe dazu Kapitel 4.2. Der Kleinbauer als gemeinsamer politischer Nenner: ein Bio-Gütesiegel 1981, die Futtermittelinitiative und die Kleinbauerninitiative.

45 A. Franc: Wie der Vorort.

46 A. Franc: Agricultural Protectionism.

47 Winterberger, Gerhard: Schweizerische Eigenart und europäische Integration, in: Schweizer Monatshefte 40/5 (1960) 456-468, 461-462. 
Damit hielt Winterberger 1961, zu Beginn seines Aufstiegs zu einem der bedeutendsten Taktgeber der Schweizer Wirtschaftspolitik der 1970er- und 80er-Jahre, die Marschrichtung fest. Diese wies eine sehr breite Übereinstimmung mit den Anliegen der kirchlich-bürgerlichen Dritte-Welt-Aktivisten auf, die sich 1970 im Bundeshaus versammelt hatten. Bereits ein Jahrzehnt zuvor hatte Winterberger ähnlich wie Paul Jolles als Leiter der Schweizer UNCTAD-Delegation - auf die aussergewöhnliche globale Verankerung der Schweizer Wirtschaft hingewiesen. ${ }^{48}$ Gerade diese starke Verankerung der Schweizer Firmen ausserhalb Europas schliesse eine Reduktion auf den europäischen Wirtschaftsraum aus:

Schliesslich spielen auch rein ökonomische Überlegungen eine Rolle. Die Übernahme des protektionistischen Aussentarifs [der EWG] und die Harmonisierung und Gleichschaltung der Wirtschafts- und Sozialpolitik würde unsere internationale Wettbewerbsstellung beeinträchtigen und die kollektivistischen Tendenzen in der Wirtschaft entsprechend verstärken. ${ }^{49}$

Keine Frage, Gerhard Winterberger, der „achte Bundesrat“ der 1970er- und 80erJahre, hatte nicht die geringste Absicht - und mit ihm ein breites Feld bürgerlicher Politiker -, der EWG beizutreten, und hielt das europäische Projekt für eine kaum weniger verachtenswerte Variante des Kollektivismus als die Sowjetunion hinter dem Eisernen Vorhang des Kalten Kriegs. Die Vertreter der Schweizer Privatwirtschaft und die Schweizer Anwälte der Dritten Welt vertraten gemeinsam einen breiten und allumfassenden Konsens: Die Schweiz sollte global handeln, nicht nur europäisch. Damit war das Thema Beitritt der Schweiz zur EWG abgeschlossen, bevor es überhaupt je wirklich zur Debatte stand. Weder die Dritte-Welt-Aktivistinnen und -Aktivisten noch die Bürgerlichen hatten vor, dem „,vorgesehenen europäischen Superstaat mit seinen explosiven Kolonial- und Grenzkonflikten“ beizutreten. ${ }^{50}$ Als die EvB im Oktober 1971 schliesslich in einer Pressekonferenz ihre kommissionierte ökonomische Studie zu einem potentiellen Beitritt der Schweiz zur EWG präsentierte, äusserte sich die Presse durchaus wohlwollend und repetierte sozusagen die Argumente, die bereits von bürgerlicher Seite wiederholt vorgebracht worden waren..$^{51}$ So schrieb etwa die Basler „NationalZeitung“: „Es ist nicht möglich, einen engeren Zusammenschluss Europas durchzuführen, ohne gleichzeitig alle anderen, nicht beteiligten Länder zu diskriminieren. “52

48 S. Misteli: Der UNCTAD-Moment, 207.

49 G. Winterberger: Schweizerische Eigenart, 462.

50 Ebd.

51 A. Wagner: EWG und Dritte Welt.

52 Henri Stranner, Kehrseite der Integration, National Zeitung, 24.10.1971. 
Was die Konferenz von 1970 nicht in den Text einbaute und nicht voraussah, war, dass die Ausfuhr subventionierter Agrarprodukte von Industriestaaten in die Entwicklungsländer zum eigentlichen Problem werden würde. ${ }^{53}$ Es war im Gegenteil so, dass sich die Konferenzteilnehmerinnen und -teilnehmer 1970 noch allen Ernstes vorstellten, dass die Entwicklungsländer rasch zu Konkurrenten würden und in der Schweiz für die sozialen Verlierer dieser neuen Konkurrenz gesorgt werden müsste. In einer Schrift, welche die EvB als neu gegründeter Verein im Rahmen der Konferenz genehmigt hatte und die sozusagen die Haltung des Vereins 1970 widerspiegelt, steht:

Ein Strukturwandel ist unabwendbar. Ferner sind Übergangsmassnahmen vorzubereiten für jene Gruppen unserer Bevölkerung und für jene Sektoren unserer Wirtschaft, die am meisten von einer grosszügigeren Importpolitik für Produkte aus Entwicklungsländern betroffen sein werden („Erklärung von Bern“, art. 5) ${ }^{54}$

Nicht nur im Jahr 1968, auch noch 1970 waren die Dritte-Welt-Aktivistinnen und -Aktivisten überzeugt davon, dass aus der Dritten bald eine Erste Welt werden würde. Nicht im Traum konnten sich die Konferenzteilnehmer von 1970 vorstellen, dass die Industrieländer so vehement darauf bestehen würden, ihre landwirtschaftlichen Produkte selbst herzustellen. Wohl ebenso wenig konnten sie sich vorstellen, wie schnell aus den vielen rohstoffexportierenden Entwicklungsländern, über die sie diskutierten, rohstoffimportierende Länder werden würden. ${ }^{55}$ Kaum ein Aspekt der Nord-Süd-Beziehung der vergangenen Jahrzehnte war überraschender und fataler als die Entwicklungen in der Landwirtschaft. ${ }^{56}$

Tatsächlich wurden Landwirtschaft und Ernährung nach $1971 \mathrm{zu}$ einem Kernthema der EvB und blieben dies bis heute. Doch das Konzept hat sich von den „Grundlagen“, die in der Konferenz von 1970 erarbeitet wurden, losgelöst. Unter Beibehaltung der ursprünglichen Haltung zur Schweizer Landwirtschaft wäre der Aufbau einer professionellen NGO nicht möglich gewesen. Zu stark, zu tief und zu emotional erwies sich die Bindung der Schweizerinnen und Schweizer an ihre Landwirtschaft. In der Schweiz erwies sich dieses Band sogar noch als viel stärker als in anderen Industrieländern, was für die kommenden Jahrzehnte die höchsten Agrarsubventionen weltweit zur Folge hatte. ${ }^{57}$

53 J. E. Stiglitz, A. Charlton: Fair Trade for All, 45.

54 SozArch, EvB, Ar 430.25.1. EvB, Anfangszeit EvB: Die Schweiz und die Entwicklungsländer, Text, der am 14.11.1970 in Bern vom Schweizer Komitee der EvB genehmigt wurde.

55 P. Bairoch: Economics and World History, 152.

56 P. Collier: The Bottom Billion, 159.

57 J. E. Stiglitz, A. Charlton: Fair Trade for All. 


\subsection{Vom christlichen Ehrenamt über Frauenarbeit zur professionellen Lohnarbeit}

Die „Erklärung von Bern“ war ein letztes Aufbäumen der reformierten Landeskirche, gleichzeitig aber auch eine Bruchstelle, die sich 1968 in der Schweiz auftat. Nachdem sich die reformierten Theologen vor 1968 noch in den herkömmlichen Gremien getroffen hatten, etwa im Rahmen der Jahrestagungen des Evangelischen Kirchenbundes, organisierte der Basler Barthianer Max Geiger im Januar 1968, sozusagen als Auftakt zu diesem ereignisreichen Jahr der Aufbrüche, ein loses Treffen ausserhalb der klassischen Strukturen im Tagungszentrum Gwatt. Zusammen mit Lukas Vischer und André Biéler lud er Gleichgesinnte ein:

Uns beschäftigt seit längerem die Frage, wie wir dem, was uns aufgetragen ist, gerecht werden können. [...] und nach dem zu fragen, was in unserem Lande und in unserer evangelischen Kirche in diesen Tagen wichtig ist. Wir denken dabei vor allem an die Beziehung zwischen reichen und armen Völkern. ${ }^{58}$

Der „Auftrag“, dem sich die drei Theologen stellten, nährte sich aus der jahrhundertealten christlichen Tradition. Gleichzeitig war er richtungsweisend für die jüngere Generation, die in den darauffolgenden Jahren auf einer scheinbar säkularen Ebene mit einem ebenso tief empfundenen „Auftrag“ „die Beziehung zwischen reichen und armen Völkern“ anzugehen suchte. Im Januar 1968 setzten die Theologen in Gwatt eine erste Erklärung auf, die deshalb in den ersten Entwürfen noch „Déclaration de Gwatt“ heisst. ${ }^{59}$ Im März 1968 lud Geiger im Namen der „Redaktionskommission“ dieser Erklärung, die sich in Gwatt gebildet hatte, $\mathrm{zu}$ einem weiteren Treffen nach Bern. ${ }^{60}$ Nach diesem Treffen, das im November 1968 stattfand, stand schliesslich die „Erklärung von Bern““ ${ }^{61}$ Dutzende bekannte Schweizer Persönlichkeiten unterschrieben sie, neben Karl Barth beispielsweise auch der Basler Philosophieprofessor und Gesellschaftskritiker Arnold Künzli, der Berner Chansonnier Mani Matter oder für die Romandie Pierre Bungener, der in

58 SozArch, EvB, Ar 430.25.1. EvB, Anfangszeit: André Biéler, Max Geiger, Lukas Vischer an „Freunde und Brüder“, „Anfang“ Dezember 1967.

59 SozArch, EvB, Ar 430.25.1. EvB, Anfangszeit: „Déclaration de Gwatt“, Projet de procédure, ohne Datum, ohne Autor.

60 SozArch, EvB, Ar 430.25.1. EvB, Anfangszeit: Redaktionskommission an „Freunde und Brüder“, „Anfang“" März 1968.

61 SozArch, EvB, Ar 430.25.1. EvB, Anfangszeit: Max Geiger an „Freunde“, 14.10.1968, Einladung zum Treffen. 
Genf gerade das Institut africain aufbaute. ${ }^{62}$ Am 10. Januar 1969 übergaben die Initianten das Manifest mit über tausend Unterschriften Bundesrat Willy Spühler, dem Vorsteher des Politischen Departements, und organisierten eine Pressekonferenz. ${ }^{63}$ Sie alle glaubten, die Bewegung im Kontext des Manifests sei „befristet“ und „politisch nicht gebunden“. ${ }^{4}$ Dabei hatte Max Geiger zunächst übersehen, dass seine Sekretärin, Frau Mundwiler, von schriftlichen Anfragen überschwemmt wurde und mit der Arbeit für die „Erklärung von Bern“ nicht nachkam. ${ }^{65}$ Daher schlug er vor, sich im Januar 1969 nochmals zu treffen. Genau ein Jahr nach dem ersten Treffen im Januar 1968 in Gwatt markierte diese Zusammenkunft vom Januar 1969 in Bern das Ende des Jahres 1968 und den Aufbruch in die Nach-68-Zeit, welche zu einer Institutionalisierung und Professionalisierung der Dritte-WeltBewegung führte. Diese griff zwar noch auf die kirchlichen Fundamente zurück, operierte jedoch losgelöst von diesen bereits auf einer säkularen, nichtgouvernementalen Ebene. So stand im Januar 1969 einerseits die Diskussion über das „weitere Vorgehen“ auf der Traktandenliste, andererseits „die Wahl eines ständigen Mitarbeiters“, zunächst einmal - in der aus heutiger Sicht rührenden Formulierung - „für die nächsten Monate“. ${ }^{66}$

Die weitere Entwicklung und die Umstände sind in den Erinnerungen des „ständigen Mitarbeiters“ bzw. der ersten Sekretärin Anne-Marie Holenstein nachzulesen. Zunächst wurde diese von Mitinitiant Hans Ruh angefragt, doch sie kannte bereits mehrere Initianten aus ihrer ökumenischen Arbeit. ${ }^{67}$ Als Katholikin und junge Mutter schien Holenstein für die unverbindliche und zunächst temporäre Sekretariatsarbeit geeignet zu sein. So sollte sie einerseits das katholische Milieu der Schweiz noch stärker ins Boot der „Erklärung von Bern“ holen, andererseits schien die Sekretariats- und Korrespondenzarbeit für eine junge Mutter, von der eigentlich erwartet wurde, dass sie gar nicht berufstätig war, passend. ${ }^{68}$ Die Führung des Sekretariats der EvB war von den Pfarrern zunächst als Frauenarbeit klassifiziert und als eine Art bezahlte Halbehrenamtlichkeit gesehen

62 SozArch, EvB, Ar 430.25.1. EvB, Anfangszeit: „Erklärung von Bern“, Entwurf, ohne Datum. Als H. P. Matter (Cambridge). Zu Künzli siehe Sidler, Roger: Arnold Künzli. Kalter Krieg und ,geistige Landesverteidigung،, eine Fallstudie (Zürich 2006).

63 A.-M. Holenstein-Hasler, R. Renschler, R. H. Strahm: Entwicklung heisst Befreiung, 23.

64 SozArch, EvB, Ar 430.25.1. EvB, Anfangszeit: Tagung in Boldern, 28.2./1.3.1969. Öffentlickeit und Entwicklungshilfe, ohne Autor.

65 SozArch, EvB, Ar 430.25.1. EvB, Anfangszeit: Peter Gessler an Frau H. Mundwiler-Flückiger, 5.7.1968.

66 SozArch, EvB, Ar 430.25.1. EvB, Anfangszeit: Max Geiger an die Mitglieder der erweiterten Redaktionskommission, 17.1.1969.

67 A.-M. Holenstein-Hasler, R. Renschler, R. H. Strahm: Entwicklung heisst Befreiung, 16.

68 Ebd. 
worden. In den Quellen wird dieses Verständnis der Ehrenamtlichkeit auch dadurch ersichtlich, dass kaum Buchhaltung erhalten ist. Dass Holenstein promovierte Germanistin war und für die Arbeit als Sekretärin sowohl überqualifiziert als auch unterbezahlt war, schien trotz der Aufbruchsstimmung von 1968 weder den Theologieprofessoren noch Holenstein aufzufallen. Die Organisation der EvB dürfte teilweise alleine entstanden sein, weil Holenstein unterfordert war und ihre Fähigkeiten einsetzte. Denn faktisch agierte sie von Beginn weg nicht als Sekretärin, sondern als unternehmerische Geschäftsführerin. Im Laufe des Jahres 1969 übernahm sie von Max Geigers Sekretärin Frau Mundwiler die Adresskartei, die im Rahmen der „Erklärung von Bern“ entstanden war. Sie entwickelte ein Konzept für die Weiterführung, verfasste Rundbriefe und vertrat die im Entstehen begriffene Organisation in den Medien. ${ }^{69}$ Und: Holenstein holte äusserst erfolgreich die katholische Innerschweiz mit ins Boot. Die Bereitschaft, mit der im Laufe des Jahres 1969 eine ganze Reihe von Innerschweizer Frauenklöstern in corpore die Erklärung unterschrieben, zeigt denn auch die Ausweitung der Bewegung von der radikalen Randgruppe um die Barthianer von 1968 auf die zwar durch die äussere Mission globalisierte, aber gesellschaftlich hochkonservative katholische Innerschweiz auf. „Das grosse Anliegen der Entwicklungshilfe schliessen wir gerne und selbstverständlich in unser tägliches Gebet ein“, schrieb etwa die Vorsteherin des Frauenklosters Maria Opferung in Zug, Schwester Maria Angelica Moos, an AnneMarie Holenstein. ${ }^{70}$ Unterschrieben hatten auch Schwestern der Frauenklöster St. Joseph Montorge in Fribourg, Maria Hilf in Altstätten, St. Klara in Stans, vom Namen Jesu in Solothurn, St. Maria der Engel in Wattwil und die Kapuzinerinnen St. Karl in Altdorf, um nur einen kleinen Teil der katholischen Unterstützung zu nennen. ${ }^{71}$ Insbesondere aber begann Holenstein, sich sofort international mit ähnlichen Bewegungen zu vernetzen. Bereits im Frühling 1970 machte sie in den Niederlanden, wo sie mit der Familie die Ferien verbrachte, einen kleinen Abstecher und nahm an einem im Rückblick bahnbrechenden Treffen holländischer Aktivisten teil, die bereits 1968 mit der Zuckerkampagne ihre EWG-Kritik lanciert hatten. ${ }^{72}$ Im Juni 1970 stand Holenstein in Kontakt zu einer ebenfalls in den Niederlanden stationierten internationalen (nichtkirchlichen) NGO namens Tax for world welfare, die sich weiter gegen den Agrarprotektionismus der Europäi-

69 SozArch, EvB, Ar 430.11.1. Bericht von Anne-Marie Holenstein an „Mitglieder“, Mitte November 1969.

70 SozArch, EvB, Ar 430.19.1. Korrespondenz EvB 1969: Frau Mutter Sr. Maria Angelica Moos, Frauenkloster Maria Opferung in Zug, an Anne-Marie Holenstein, 31.10.1969.

71 SozArch, EvB, Ar 430.19.1. Korrespondenz EvB 1969.

72 SozArch, EvB, Ar 430.10.1. Anne-Marie Holenstein an Novib, 2.1.1970. Siehe dazu auch Dam, Peter Van: Moralizing Postcolonial Consumer Society. 
schen Wirtschaftsgemeinschaft einsetzte und auf das Beispiel Zucker hinwies. ${ }^{73}$ Gleichzeitig stellte sie weitere Frauen an, die ihr nicht nur bei der Kinderbetreuung, sondern auch bei der Sekretariatsarbeit zu Hilfe gingen. ${ }^{74}$ Unter dem Deckmantel der Frauenarbeit war eine bedeutende Schweizer NGO geboren. Im August 1970, nachdem Anne-Marie Holenstein bereits ein Jahr lang an der Arbeit war, wandte sich Max Geiger ein weiteres Mal an die Unterzeichner der „Erklärung von Bern“, und erklärte:

[Es] drängt sich [...] die Gründung einer ,Vereinigung zur Förderung der Erklärung von Bern` auf; ihre Statuten sollen seinem Aktionsausschuss erlauben, öffentlich im Namen der Mitglieder Stellung zu nehmen. ${ }^{75}$

Im November 1970 wurden die Statuten genehmigt, womit ein Verein entstanden war. ${ }^{76}$ Die Barthschen Aktivisten hatten sich der Institutionalisierung ohne Enthusiasmus gebeugt. Dies ganz im Sinne eines im Vorjahr entstandenen Chansons des Berner Chansonniers Mani Matter, der einer der ersten Unterzeichner der Erklärung war:

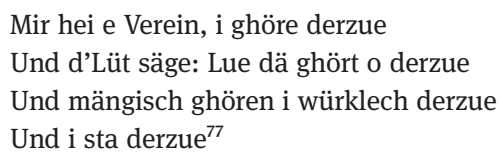

Widerwillig und ohne Absicht hatten die Erstunterzeichner um Max Geiger einen Verein gegründet. Doch eigentlich war für sie die Aktion von 1968 bereits wieder vorbei - den Aufbau einer Institution hatten sie weder vorgesehen noch hatten die Erstunterzeichner aufgrund ihres Alters und der ihnen zur Verfügung stehenden Zeit Kapazitäten dafür. Zunächst bestand bis 1971 ein inoffizielles Komitee, das sich aus den ersten Verfassern des Manifests gebildet hatte. ${ }^{78}$ Die letzte Sitzung des ursprünglichen Initiativkomitees fand im Juni 1971 statt, allerdings nahm kaum noch jemand teil. Biéler hatte aus Gesundheitsgründen bereits demissioniert, Geiger hegte ebenfalls Rücktrittsabsichten. ${ }^{79}$ Anne-Marie Holenstein kam

73 SozArch, EvB, Ar 430.11.1. Mappe 1: Korrespondenz mit tax of [sic!] worldwelfare 1970, Bericht über die Common Agricultural Policy, Juni 1970.

74 A.-M. Holenstein-Hasler, R. Renschler, R. H. Strahm: Entwicklung heisst Befreiung, 41-42.

75 SozArch, EvB, Ar 430.11.1. Deutschschweizer-Komitee: Rundbrief 3, August 1970.

76 SozArch, EvB, Ar 430.11.1. Deutschschweizer-Komitee: Statuten vom 14.11.1970.

77 Matter, Mani; Vatter, Ben; Zurbriggen, Silvan: Mani Matter - Liederbuch (Basel 2015) 96.

78 A.-M. Holenstein-Hasler, R. Renschler, R. H. Strahm: Entwicklung heisst Befreiung, 53.

79 SozArch, EvB, Ar 430.10.1. Protokoll der letzten Sitzung des Initiativkomitees, 18.6.1971. 
die undankbare Aufgabe zu, einen neuen Präsidenten zu suchen, worauf sie Anfragen an verschiedene Erstunterzeichner schickte. Sie erhielt einen ganzen Stapel Entschuldigungsbriefe. Niemand hatte Zeit, keiner mochte die administrative Arbeit machen. Bezeichnend war etwa das Schreiben des Berner NydeggPfarrers Kurt Marti:

Ich hatte schon bisher ein schlechtes Gewissen, weil ich da mehr am Rande noch mitschwamm, ohne viel Nützliches beitragen zu können. Aber jetzt ist ja wohl der Moment, um mich [...] in aller Form und mit Anstand aus dem Komitee zu verabschieden. ${ }^{80}$

Lange konnte Anne-Marie Holenstein schlicht keinen Anwärter auf das Amt des Präsidenten der EvB finden, bis sich schliesslich der Genfer Bankangestellte Georges Rossier fand. ${ }^{81}$ Dies illustriert, wie die NGO zu einer Institution der Angestellten geworden war, welche die Organisation und ihre Entwicklung prägten und ihr in der Öffentlichkeit ein Gesicht gaben. Die - ehrenamtliche - Arbeit im Aufsichtsorgan über die NGO, die offiziell als Verein konstituiert war, trat von da an stark in den Hintergrund.

Während die arrivierten Theologen und Pfarrer nichts mit dem neuen Verein anfangen konnten, stellte dessen Gründung doch ein Sprungbrett für die Frauen und die „Jugendfraktion“ bereit. Die fortdauernde „Frauenarbeit“ im Verein illustriert dies. Alle ersten Angestellten waren Frauen und zudem junge Mütter. ${ }^{82}$ Die Jahre nach 1968 waren für Frauen in der Schweiz eine bedeutende Aufbruchzeit. 1971 erhielten sie das eidgenössische Stimmrecht, 1972 publizierte das „Magazin“ des „Tages Anzeigers“ unter der Ägide von Laure Wyss erstmals eine anonyme Umfrage bei Frauen, in welcher deren unbefriedigende Stellung im Arbeitsmarkt als Schwerpunktthema dargestellt wurde. ${ }^{83}$ Die Arbeit beim Aufbau der NGO war für die ersten Mitarbeiterinnen ein empowerment, das diese selbst überraschte. Es erlaubte ihnen, trotz kleiner Kinder eine ihnen am Herzen liegende Arbeit von zuhause aus zu verrichten und erst noch dafür bezahlt zu werden. Wie froh die ersten weiblichen Angestellten der EvB waren, überhaupt für ihre Arbeit bezahlt zu werden, zeigt sich daran, dass Anne-Marie Holenstein auf einen Teil ihres Lohnes verzichtete, damit Regula Renschler eingestellt werden konnte. ${ }^{84}$ Weder in den Quellen noch in den autobiografischen Berichten von Anne-Marie Holenstein und Regula Renschler wird sichtbar, dass diese je ihren

80 SozArch, EvB, Ar 430.10.1. Kurt Marti an Anne-Marie Holenstein, 8.7.1971.

81 A.-M. Holenstein-Hasler, R. Renschler, R. H. Strahm: Entwicklung heisst Befreiung, 69.

82 Ebd., 41-42.

83 Ergebnis einer Umfrage [Titel der Ausgabe], Tages-Anzeiger Magazin, 11.11.1972.

84 A.-M. Holenstein-Hasler, R. Renschler, R. H. Strahm: Entwicklung heisst Befreiung, 123. 
Lohn neu verhandelt oder eine Erhöhung gefordert hätten. Gleichzeitig mit Renschler wurde Rudolf Strahm 1974 als leitender Sekretär eingestellt und damit zum Vorgesetzen der beiden Frauen, obwohl diese älter und zudem im Gegensatz zu Strahm promoviert waren. ${ }^{85}$ Strahm beriet seit 1970 das Komitee der EvB in Wirtschaftsfragen. ${ }^{86}$ Nach 1970 nahm er immer wieder als Gast an Sitzungen teil und berichtete von seinen Aktivitäten in anderen, ehrenamtlichen Gruppierungen, etwa in der Arbeitsgruppe Schweiz-Dritte Welt. ${ }^{87}$ Nach seinem Stellenantritt verfolgte Strahm als erstes Ziel die Rationalisierung und Professionalisierung des NGO-Betriebs. ${ }^{88}$ Letztere wurde auch möglich durch einen Know-how-Transfer von der althergebrachten Basler Mission zur EvB. Wie bedeutend der Erfahrungsschatz der 150-jährigen Basler Mission für die noch junge EvB war, sollte Jacques Rossel beiläufig in seinen Memoiren erwähnen:

Ich wurde Mitglied [der EvB] und später Interimpräsident. Zusammen mit Rudolf Strahm habe ich das Sekretariat der ,Erklärung von Bern' reorganisiert, und er hat dann die Leitung übernommen. Es gelang mir, einige Mitglieder dazu zu bringen, 3\% ihres Einkommens zu geben, um ihm [sic!] einen angemessenen Lohn zu garantieren. ${ }^{89}$

Rossel hat mit seiner Unterschrift unter die „Erklärung von Bern“ nicht nur moralisches Kapital an die spätere NGO EvB übertragen, er wirkte auch handfest beim Aufbau professioneller Strukturen mit, nachdem sein Vorgänger, der Theologieprofessor Max Geiger, es zunächst über ein Jahr lang versäumt hatte, überhaupt einen offiziellen Verein zu gründen. So kam es zwischen der Basler Mission und der EvB zu einem Know-how-Transfer im organisatorischen Bereich, der nichts mit dem eigentlichen Auftrag der NGO zu tun hatte.

Diese Professionalisierung wurde überhaupt erst möglich, weil der Passus über die Drei-Prozent-Spende in der Erklärung sowie die ehrenamtliche Arbeit der ersten Generation einen Spendenfluss auslösten. ${ }^{90}$ Dies wiederum rief geradezu nach einer Institutionalisierung. Ursprünglich hatten die Initianten der Erklärung eine Liste mit Bankverbindungen der klassischen Schweizer Hilfswerke beigelegt und waren davon ausgegangen, dass jene Personen, die ihren Namen unter die Drei-Prozent-Spende gesetzt hatten, dieses Geld an eines der aufgelisteten Hilfs-

85 Ebd., 113.

86 Ebd., 45.

87 SozArch, EvB, Ar 430.10.1. PV de la séance du comité executif, 6.3.1971.

88 A.-M. Holenstein-Hasler, R. Renschler, R. H. Strahm: Entwicklung heisst Befreiung, 123.

89 J. Rossel: Leben in ökumenischer Weite, 294. Die Löhne der beiden Frauen werden nicht angesprochen.

90 SozArch. EvB, Ar 430.10.2. Schweizer Komitee 1974-77: Rechnung 1974, Budget 1975. 
werke überweisen würden. Möglich, dass Frau Mundwiler, Max Geigers Sekretärin, den Grundstein zur Schaffung einer der bedeutendsten Schweizer NGOs legte, indem sie ihren Chef auf den praktischen Aspekt der auflaufenden Druckkosten hinwies und ein Konto bei der Basellandschaftlichen Kantonalbank einrichtete. ${ }^{91}$ Auf jeden Fall wandte sich die Redaktionskommission bereits im Dezember 1968 an die Unterzeichnenden:

Wir ersuchen Sie deshalb höflich, einen von Ihnen frei zu bestimmenden Teil Ihrer 3\% für unsere Unkostendeckung bereitzustellen. Wie aus unserer Erklärung hervorgeht, stellt die Meinungsumbildung in den reichen Ländern eine der ersten Bedingungen für die Entwicklung der armen Länder dar. ${ }^{92}$

Was die Redaktionskommission in ihrem ersten Spendenaufruf noch etwas ungelenk als „Meinungsumbildung“ bezeichnete, sollte unter dem Begriff „Bewusstseinsbildung“ zur Kernkompetenz der NGO werden. Im Februar 1969 wandte sich Max Geiger wiederum an die „deutschsprachigen Mitglieder der Redaktionskommission“, informierte sie, dass Anne-Marie Holenstein nun die Sekretariatsarbeit übernehmen würde, und bat sie, „einen möglichst hohen Betrag Ihrer eigenen 3\% im laufenden Jahr 1969 zugunsten unserer Unkosten zu verwenden“. ${ }^{93}$ Im November 1969 zahlten auch Hilfswerke, die bis anhin von den Spenden im Rahmen der EvB profitiert hatten, Beiträge an das EvB-Sekretariat, so etwa die Basler Mission. ${ }^{94}$ Da die EvB bis November 1970 kein Verein war und auch danach erst langsam in die Strukturen eines Vereins hineinwuchs, sind in den Akten kaum Buchhaltungsunterlagen vorhanden. Die Geldfrage wurde noch bis in die 1980er-Jahre nicht ernst genommen, wie Al Imfeld 1983 kommentierte: „Umgang mit Geld ist für idealistische Gruppen stets ein heikler Punkt. Sehr oft überfordern sie sich, indem sie Eigengesetzlichkeiten nicht ernst nehmen. "95 Erst Schulden von über 80000 Franken sollten Mitte der 1980er-Jahre zur definitiven Professionalisierung der EvB auch in finanzieller Hinsicht führen. ${ }^{96}$ Doch am

91 SozArch, EvB, Ar 430.25.1. EvB, Anfangszeit: Redaktionskommission an Unterzeichner, 18.12. 1968.

92 SozArch, EvB, Ar 430.25.1. EvB, Anfangszeit: Redaktionskommission an Unterzeichner, 18.12. 1968.

93 SozArch, EvB, Ar 430.25.1. EvB, Anfangszeit: Max Geiger an die „deutschsprachigen Mitglieder der Redaktionskommission“, 14.2.1969.

94 SozArch, EvB, Ar 430.19.1. Korrespondenz EvB 1969: H. Röthlisberger, Referent für Heimatfragen, Basler Mission, an Anne-Marie Holenstein, 19.11.1969.

95 Al Imfeld, Die „Erklärung von Bern“ in der Krise, Wochenzeitung, 16.9.1983.

96 Siehe dazu Kapitel 5.1. Die Professionalisierung der Fair-Trade-Bewegung. Siehe auch K. J .Kuhn: Entwicklungspolitische Solidarität, 419. 
Anfang, zu Beginn der 1970er-Jahre, wurde die EvB mit Spenden derart gut bedacht, dass sie es sich leisten konnte, die Finanzen „,idealistisch“, sprich gar nicht anzupacken. In den Unterlagen sind einzig eine genauere Vereinsrechnung von 1974 sowie ein Budget für das Jahr 1975 vorhanden. Daraus geht hervor, dass die EvB in der deutschen Schweiz in beiden Jahren über 100000 Franken an Spendengeldern einnahm. Dazu kam der Verkauf von Büchern der EvB, die hauptsächlich aus der Feder von Rudolf Strahm stammten. Dieser Posten wurde für das Jahr 1975 mit einem Betrag von 20000 Franken budgetiert. ${ }^{97}$ Das dreiköpfige Sekretariat finanzierte sich nach 1974 hauptsächlich aus Geldern, die im Rahmen der Drei-Prozent-Spende von den Unterzeichnern eingingen.

Hier ist zu erwähnen, dass die EvB bereits vor ihrer Gründung durch André Biéler in der Romandie ebenso stark verankert war wie in der Deutschschweiz. So nahm in der Romandie etwa um 1970 der Sekretär François de Vargas seine Arbeit auf. ${ }^{98}$ Die beiden Sekretariate operierten unabhängig voneinander und generierten ihre eigenen Spenden. So bildeten sich im Oktober 1971 denn auch zwei Komitees, ein Deutschschweizer und ein Westschweizer Komitee. Zum Komitee in der Romandie gehörten etwa der Genfer Politologe Gilbert Rist (`1938), der 1985 Professor am IUED Genf wurde, oder der junge Deutschschweizer Student Mattias Meyer, der später beim eidgenössischen Staatssekretariat für Wirtschaft die Abteilung Entwicklungszusammenarbeit aufbauen würde. ${ }^{99}$

Als die „Erklärung von Bern“ 1968 publiziert wurde, waren die meisten Unterzeichner ehrenamtlich für die Dritte Welt tätig. Sie verpflichteten sich, drei Prozent ihres Einkommens, das sie in „normalen“ Berufen erzielten, für die Dritte Welt zu spenden. Die „Erklärung von Bern“ war nicht nur der Anfang einer neuen professionellen NGO, sondern gleichzeitig auch die letzte Demonstration der „alten Garde“, des wohlhabenden, sozial engagierten Bürgertums, für das wohltätiges Engagement eine Selbstverständlichkeit war. Doch die EvB forderte vom Bund eine Professionalisierung bezüglich des Umgangs mit der Dritte-Welt-Thematik. Sie verlangte im Manifest mehr Steuergelder für Entwicklungshilfe, ein

97 SozArch. EvB, Ar 430.10.2. Schweizer Komitee 1974-77: Rechnung 1974, Budget 1975.

98 SozArch, EvB, Ar 430.11.1. Mappe 3: Korrespondenz Sekretariat Romandie und Deutschschweiz.

99 SozArch, EvB, Ar 430.10.1. Schweizer Komitee 1970-1973. Die offizielle Archivierung der Akten des Westschweizer Komitees beginnt jedoch erst im Jahr 1975. Siehe dazu SozArch, EvB, Ar 430.12.1. Comité romand 1975-1983. Zur Biografie Rists siehe auf der Webseite des heutigen Graduate Institute in Genf

http://graduateinstitute.ch/files/live/sites/iheid/files/sites/admininst/shared/cv-professors/CVGilbert.Rist.pdf (10.4.2018). Zu Meyer siehe etwa seine Publikation Bürgi, Elisabeth; Meyer, Matthias; Ferrarini, Benno: Agricultural Exports as Engine of Growth for Developing Countries? A Case Study on International Trade in Tobacco (Bern 2003). 
vom Bund finanziertes Institut für Studien zur Dritten Welt und die Aufnahme der Dritten Welt in den Lehrplan der Schulen. Im Rückblick lässt sich kommentieren, dass die EvB die Geister, die sie rief, nicht mehr loswurde. Was in den Jahren nach der Erklärung von 1968 folgte, war ein starker Anstieg der öffentlichen Entwicklungshilfe. Die Erhöhung der öffentlichen Entwicklungshilfe der Schweiz lässt sich leicht in Zahlen fassen, sie stieg in der hier untersuchten Periode zwischen 1964 und 1984 von 39,7 Millionen auf 670,9 Millionen Franken und damit um das Siebzehnfache. ${ }^{100}$ Ausserdem wurde die spätere Eidgenössische Direktion für Entwicklung und Zusammenarbeit (DEZA) rasant ausgebaut und bot zahlreichen jungen Entwicklungsexperten gut bezahlte Stellen in der Bundesverwaltung. Die Arbeit des Missionars, abenteuerlich, oft lebensgefährlich und eine lebenslange Berufung, ging an Angestellte und gar an eidgenössische Beamte über. ${ }^{101}$ Max Frisch hat mit seinem Romantitel „Homo Faber“ 1957 diesen neuen Typus des Entwicklungshelfers benannt. ${ }^{102}$

Im Nichtregierungsbereich verlief diese Entwicklung ähnlich. Anne-Marie Holenstein setzte sich stark für den Aufbau einer Organisationsstruktur ein und übernahm dabei wie erwähnt die Rolle einer aktiven Geschäftsführerin, die auch gegenüber dem Vorstand immer wieder mahnte, das Ziel eines realisierbaren Organisationsaufbaus nicht aus den Augen zu verlieren. ${ }^{103}$ Was Holenstein nicht leistete, war eine fundierte Auseinandersetzung bezüglich der Umsetzung des handelspolitischen Schwerpunkts der EvB, wie er auf der Konferenz von 1970 noch stärker herausgearbeitet worden war. Dass zunächst vor allem Theologen ohne ökonomisches oder juristisches Fachwissen dem Vorstand angehörten - und nicht etwa Christoph Eckenstein, der 1974 bereits an einer Krankheit sterben sollte, - begünstigte dieses Manko. Holenstein war Germanistin und hatte weder die Zeit, sich mit wirtschaftswissenschaftlicher Theorie und ihrer juristischen Umsetzung $\mathrm{zu}$ beschäftigen, noch hätte sie sich dies zugetraut. ${ }^{104}$ Holenstein schreibt:

100 Siehe dazu auf der Webseite der Eidgenössischen Direktion für Entwicklung und Zusammenarbeit (Deza) „Entwicklung der öffentlichen Entwicklungshilfe (APD) und der privaten Spenden der NGOs der Schweiz 1960-2015 (Mio. Fr.)“, (https://www.eda.admin.ch/deza/de/ho me/aktivitaeten_projekte/zahlen_und_statistiken/statistische-tabellen.html) (22.9.2017).

101 R. Lenzin: Schweizer im kolonialen und postkolonialen Afrika.

102 Frisch, Max: Homo faber. Ein Bericht (Frankfurt am Main 1957).

103 SozArch, EvB, Ar 430.10.2. Anne-Marie Holenstein: Ueberlegungen betr. Entwicklungspolitische Verfassungsinitiative, 16.4.1975.

$104 \mathrm{Zu}$ dieser Verunsicherung siehe auch Holenstein, Anne-Marie: Jute statt Plastik, in: MigrosGenossenschafts-Bund (Hg.): M-Frühling. Vom Migrosaurier zum menschlichen Mass (Bern 1980) 185-198, 186. 
Ich hatte neben der Sekretariatsleitung im Halbtagsjob, der Mitarbeit in der Redaktion der von Frauen neu gegründeten ökumenischen Zeitschrift ,Schritte ins Offene ‘ und der jungen Familie weder Zeit noch Energie, mich mit den Dependenztheorien und dem Zentrum-Peripherie-Modell auseinanderzusetzen. ${ }^{105}$

Die Diskrepanz zwischen dem Postulat der Notwendigkeit von handelspolitischen Umstellungen und dem Begreifen und Umsetzen wirtschaftswissenschaftlicher Grundlagen bildet eine Kontinuität in der Bewegung um die „Erklärung von Bern“. Bereits Punkt 4 der Erklärung, in dem die Handelsbeziehungen angesprochen werden, ist holperig formuliert; die Verfasser machten es sich einfach, indem sie auf die UNCTAD verwiesen. ${ }^{106}$ Der Bericht der Konferenz von 1970 hingegen fiel fachlich korrekter und klarer aus, was auf die Mitarbeit des Ökonomen Strahm und der beiden Juristen Preiswerk und Eckenstein, die beide seit Jahren als Experten der Nord-Süd-Handelspolitik agierten, zurückzuführen ist. ${ }^{107}$

Als der Aufbau der Organisation EvB in den Händen einer Germanistin lag, fiel das wirtschaftswissenschaftliche Fachwissen wie kurze Zeit zuvor bei den Theologen unter den Tisch. Dies bedeutet nicht, dass nicht die gesamte Bewegung bis Mitte der 1970er-Jahre tief überzeugt war von der Notwendigkeit ökonomischer Strukturveränderungen. In der Frage, wie diese nun genau umgesetzt werden sollte, kamen sowohl die Initianten der „Erklärung von Bern“ wie auch die erste Sekretärin sprachlich schnell ins Schleudern und behalfen sich damit, abwechslungsweise auf die UNCTAD, auf ,unsere kompetentesten Volkswirtschafter" oder auf Rudolf Strahm zu verweisen. ${ }^{108}$ So etwa in einem Brief, den AnneMarie Holenstein im Mai 1971 an die Aussenwirtschaftskommisssion der Bundesversammlung schrieb und diese bat, die Vorlage des Bundesrates über die Zollpräferenzen zur Annahme zu empfehlen. ${ }^{109}$ In der Beilage des Briefes befand sich ein Separatdruck von Rudolf Strahms Diplomarbeit über den effektiven Zollschutz der Schweiz gegenüber den Entwicklungsländern. ${ }^{110}$ Anstatt sich selbst in die Thematik einzuarbeiten, verwies Holenstein auf Strahms Text. Strahm beriet das Komitee ab 1970 in ökonomischen Fragen, sozusagen als

105 A.-M. Holenstein-Hasler, R. Renschler, R. H. Strahm: Entwicklung heisst Befreiung, 35.

106 https://www.publiceye.ch/fileadmin/files/documents/Ueber_uns/EvB_1968_0.pdf (28.3. 2018). Siehe auch SozArch, EvB, Ar 430.25.1. EvB, Anfangszeit: Die Erklärung von Bern, März 1968. 107 H. Schmocker, M. Traber: Schweiz-Dritte Welt.

108 Punkt 6, Die Erklärung von Bern, https://www.publiceye.ch/fileadmin/files/documents/Ue ber_uns/EvB_1968_0.pdf (28.3.2018). Siehe auch SozArch, EvB, Ar 430.25.1. EvB, Anfangszeit: Die Erklärung von Bern, März 1968.

109 SozArch, EvB, Ar 430.10.1. Mappe 1: Anne-Marie Holenstein an die Aussenwirtschaftliche Kommission des National- und Ständerates, 13.5.1971.

110 R. H. Strahm: Effektiver Zollschutz. 
Schüler oder Vertreter von Preiswerk und Eckenstein. ${ }^{111}$ Eine tatsächliche Ausbildung der Initianten des Manifests, geschweige denn der weiblichen Sekretärin, fand jedoch nicht statt, so dass der Verweis auf „kompetente Volkswirtschafter“ zum Regelfall wurde. ${ }^{112}$ Dies ist umso erstaunlicher im Hinblick auf den Schwerpunkt der „Bewusstseinsbildung“ der Bewegung.

Die erste Priorität, kaum war die Organisation gegründet, schien - und dies sollte eine Konstante bleiben - die Beeinflussung der öffentlichen Meinung, die „Bewusstseinsbildung“, ursprünglich „Meinungsumbildung“ genannt: ${ }^{113}$

Wir leiden darunter, dass es uns kaum gelingt, mit unseren politischen Bemühungen die Grenzen der eigenen Kreise zu überspringen und zu einem wesentlichen Faktor der öffentlichen Meinung unseres Landes zu werden. ${ }^{114}$

So stand es etwa in einer Mitte der 1970er-Jahre verfassten Standortbestimmung. ${ }^{115} 1971$ hatte der Bund das Zollpräferenzsystem für Entwicklungsländer eingeführt, gleichzeitig mit einer rasanten Erhöhung der eidgenössischen Entwicklungshilfe. So kam der Bund der NGO sozusagen zuvor und erfüllte die Forderungen von 1968 schneller und erst noch so umfangreich, dass die kleine NGO davon überwältigt wurde und mit der rasanten Entwicklung kaum Schritt halten konnte.

Von aussen betrachtet stellt sich die Frage, weshalb intern nie diskutiert wurde, inwiefern es eine solche NGO überhaupt noch brauchte. Mit der Einstellung eines leitenden Sekretärs 1974 ging es denn auch darum, ein klares Betätigungsfeld für den Verein zu definieren. Die folgenden Jahre waren stark geprägt von einer mäanderartigen Suche nach einem klaren Auftrag. So stark die Aktivisten empfanden, einen „Auftrag“ zu haben, so schwierig war es, diesen auf dem Boden der organisatorischen Realität zu definieren. ${ }^{116}$ Mitte der 1970er-Jahre lässt

111 A.-M. Holenstein-Hasler, R. Renschler, R. H. Strahm: Entwicklung heisst Befreiung, 45., R. Preiswerk, G. Rist: A contre-courants.

112 Punkt 6, Die Erklärung von Bern, https://www.publiceye.ch/fileadmin/files/documents/Ue ber_uns/EvB_1968_0.pdf (28.3.2018). Siehe auch SozArch, EvB, Ar 430.25.1. EvB, Anfangszeit: Die Erklärung von Bern, März 1968.

113 SozArch, EvB, Ar 430.25.1. EvB, Anfangszeit: Redaktionskommission an Unterzeichner, 18.12.1968.

114 SozArch, EvB, Ar 430.10.2. Magdalena Rutz: Einige Ueberlegungen zur Situation der „Erklärung von Bern“, undatiert, vermutlich Mitte der 1970er-Jahre.

115 SozArch, EvB, Ar 430.10.2. Magdalena Rutz: Einige Ueberlegungen zur Situation der „Erklärung von Bern“, undatiert, vermutlich Mitte der 1970er-Jahre.

116 SozArch, EvB, Ar 430.25.1. EvB, Anfangszeit: André Biéler, Max Geiger, Lukas Vischer an „Freunde und Brüder“, „Anfang“ Dezember 1967. 
sich denn auch gut der graduelle Übergang von der ehrenamtlichen Bewegung zu einer Angestelltenorganisation beobachten. Auch 1975 trafen sich die Aktivisten der EvB wie frühere Male zur Tagung in Gwatt. Aus den Akten wird ersichtlich, dass dort eine freiwillige Basisbewegung um Roy Preiswerk mit der Sekretärin Anne-Marie Holenstein aneinandergeriet, die den Blickwinkel der organisatorischen Umsetzbarkeit und Effizienz hatte. ${ }^{117}$ Bereits seit sechs Jahren war AnneMarie Holenstein faktisch Geschäftsführerin einer wachsenden Organisation. Im Kontext einer losen Tagung einer Basisbewegung wurde sie „gegen [ihren] Willen beinahe in die Rolle eines Opinion leaders der Opposition gedrängt““. ${ }^{118}$ Während sich Holenstein 1975 noch als Teil einer uneinigen Basisbewegung verstand, war dieses Oppositionsverhältnis bereits Ausdruck einer Haltung von professionellen Angestellten der NGOs, wie sie in diesen Jahren in zahlreichen NGOs in Europa vorkam. ${ }^{119}$ Holenstein hatte sich an der Tagung in Gwatt gegen die Lancierung einer eidgenössischen entwicklungspolitischen Initiative gewehrt. Diese hätte für das geschäftsführende Dreierteam einen massiven Arbeitsaufwand ohne kurzfristige Resultate gebracht, und insbesondere hätte diese Initiative das Profil der NGO nicht geschärft. Stattdessen skizzierte Holenstein als Alternative sozusagen die Agenda einer entwicklungspolitischen NGO:

Anstelle einer Initiative plädiere ich für politische Aktionen mit enger begrenzter Zielsetzung. Sie sollten bezüglich der Zentrum-Peripherie-Problematik Modellcharakter haben und alternativ zur heutigen Politik eine Politik der Solidarität demonstrieren. Sie sollten dieselben Lernprozesse auslösen und dieselben politischen Ziele anvisieren wie die geplante Initiative, hätten aber folgende Vorteile:

- Kurzfristiges Reagieren auf aktuelle Ereignisse (Beispiele: Asylpolitik, Energiekonferenz, United Fruit-Skandal etc.

- Schaffen von Ereignissen (vergl. unten zu UNCTAD IV)

- Angepasste Strategien für verschiedene Adressaten (Parlament, Schweizer Multis, Konsumenten, Gewerkschaften, Kirchen etc.)

- Der Aufwand kann in zeitlicher und personeller Hinsicht eher limitiert werden als bei einer Initiative, die uns jahrelang beanspruchen und damit Kräfte binden würde, die für kurzfristige Aktionen gebraucht werden.

- Organisationen können ad hoc zusammenarbeiten. Kein Zwang zu ,geschlossener Front ${ }^{120}$

117 R. Preiswerk, G. Rist: A contre-courants, 54.

118 SozArch, EvB, Ar 430.10.2. Anne-Marie Holenstein: Ueberlegungen betr. Entwicklungspolitische Verfassungsinitiative, 16.4.1975.

119 M. Hilton: Politics of Expertise, 254.

120 SozArch, EvB, Ar 430.10.2. Anne-Marie Holenstein: Ueberlegungen betr. Entwicklungspolitische Verfassungsinitiative, 16.4.1975. 
Wie Holenstein klar festhielt, ging es darum, den zeitlichen und personellen Aufwand geschickt zu nutzen. Zudem war ihr klar, dass die NGO agil sein musste und sich zu aktuellen Themen äusserte, um im Gespräch zu bleiben sowie ihre Aktionen auf Zielgruppen zu fokussieren, je nach Möglichkeit in Zusammenarbeit mit anderen NGOs. Im Folgenden entwarf Holenstein ein Beispiel einer solchen politischen Aktion: Sie hielt fest, wie eine „Begleitung“ von internationalen Konferenzen durch NGOs aussehen könnte. ${ }^{121}$

Wir sehen hier also, dass die NGOs sozusagen im Fahrwasser der internationalen Organisationen und ihrer Konferenzen ihre professionelle Organisation aufbauten und in der „kritischen Begleitung“ der offiziellen Veranstaltung im Rahmen von Gegenveranstaltungen oder Rahmenprogrammen ein Geschäftsmodell entwickelten. Holenstein hatte im Jahr zuvor, 1974, am Begleitprogramm der Konferenz der Ernährungs- und Landwirtschaftsorganisation der Vereinten Nationen (FAO) in Rom teilgenommen, das unter anderem von der britischen Oxfam orchestriert wurde. ${ }^{122}$ Für die vierte Konferenz der UNCTAD, die im Mai 1976 in Nairobi stattfinden sollte, schlug sie nun ein derartiges Modell vor:

Beispiel eines möglichen Projekts: UNCTAD IV

- Informationsphase, beginnend im Herbst 1975. Einzelne Traktanden der Konferenz werden popularisiert (z. B. durch Konsumentenaktionen, Schulmaterial etc.)

- Oeffentliches Hearing: Schweiz-UNCTAD IV

- Kleine Anfrage im Parlament betr. Zusammensetzung und Vorbereitung der Schweizer Delegation. Frage nach deren Direktiven. Ev. parallel Briefkampagne. Proklamation unserer Forderungen für eine schweiz. Politik der Solidarität an der UNCTAD IV.

- Während der Konferenz organisierter Feed-back (Modell: Telephonkampagne in Kanada während der Welternährungskonferenz).

- Nach der Konferenz öffentliche Evaluation. ${ }^{123}$

Wie das Quellenzitat aufzeigt, bezog sich Holenstein auf ihre Beobachtungen an der FAO-Konferenz in Rom im Jahr zuvor. Auch kam sie in ihrem Vorschlag mit Ideen, die zu den Hauptpfeilern der NGO-Tätigkeit werden würden: den Konsumentenaktionen und der Verbreitung von Schulmaterial. Holenstein nannte dies „Popularisierung“. Sie schlug hier somit eine Vereinfachung vor, ein Herausgreifen einzelner Punkte und die Vermittlung dieser vereinfachten Punkte an

121 SozArch, EvB, Ar 430.10.2. Anne-Marie Holenstein: Ueberlegungen betr. Entwicklungspolitische Verfassungsinitiative, 16.4.1975.

122 Bodleain Library, Oxford, Oxfam archive, MS.Oxfam. PUB/528 - 9, PAN issues.

123 SozArch, EvB, Ar 430.10.2. Anne-Marie Holenstein: Ueberlegungen betr. Entwicklungspolitische Verfassungsinitiative, 16.4.1975. 
Konsumentinnen und Konsumenten sowie an Kinder. Möglich, dass Holenstein von der florierenden Schulabteilung der britischen Oxfam wusste, die Schulmaterial herstellte, das von Mitarbeiterinnen und Mitarbeitern der Organisation an Schulen zur Durchführung von Veranstaltungen genutzt wurde. ${ }^{124}$ Sicher jedoch haben die Aktivisten in britischen und holländischen NGOs, die - anders als Schweizer Aktivisten - nicht die Möglichkeit einer Volksinitiative hatten, seit 1968 einen nichtgouvernementalen Kampagnenstil vorgegeben, den Holenstein beobachtet hatte und nun für die EvB vorschlug. ${ }^{125}$

\subsection{Die internationale Vernetzung der Erklärung von Bern}

Der Ökumenischen Rat der Kirchen in Genf sowie die Schweizer Landeskirchen verankerten die Initianten der „Erklärung von Bern“ und ihre jüngeren Mitstreiter in einem bereits bestehenden globalen Netzwerk, das durch die Ökumene in der Nachkriegszeit gestärkt und aktiviert worden war. In diesem Netzwerk bewegte sich auch die Basler Mission, die sozusagen eine frühe, religiöse NGO ist, die schon damals nicht nur im Süden wirkte, sondern mit anderen europäischen und US-amerikanischen missionarischen Einrichtungen eng vernetzt war. Die EvB war in den ersten Jahren sozusagen Juniorpartner dieses internationalen Netzwerkes. Als die EvB nach 1969 in eine stets offizieller werdende Organisation überging, wuchs sie auf dem Humus des bereits bestehenden globalen Netzwerks, das sich mit dem Nord-Süd-Handel befasste. Das internationale Netzwerk der säkularen NGOs war zu dieser Zeit erst im Aufbau begriffen. 1962 gehörte unter anderem der Oxfam-Direktor Lesley Kirkley zu den Gründern des International Council of Voluntary Agencies. ${ }^{126}$ Diese internationale Organisation der NGOs mit Sitz in Genf sollte jedoch nicht die Schlagkraft entwickeln, die sich Kirkley erhofft hatte. ${ }^{127}$ Doch die informelle Vernetzung über die Aktivistinnen und Aktivisten selbst funktionierte über bereits bestehende Kanäle. So lud die niederländische Gruppierung Novib, die Mitte der 1950er-Jahre unter anderem vom Entwicklungsökonomen Jan Tinbergen gegründet worden war, die Aktivistinnen und Aktivisten der EvB in Genf im Frühling $1970 \mathrm{zu}$ ihrem Treffen nach Egmond aan Zee ein. ${ }^{128}$ Die

124 M. Black: Oxfam the First 50 Years, 102.

125 SozArch, EvB, Ar 430.10.2. Anne-Marie Holenstein: Ueberlegungen betr. Entwicklungspolitische Verfassungsinitiative, 16.4.1975.

126 https://www.icvanetwork.org/ (8.1.2018).

127 M. Black: Oxfam the First 50 Years, 101.

128 Siehe dazu die Webseite der niederländischen Oxfam, die Novib weiterführt (http://www.ox famnovib.nl/wat-wij-doen/over-oxfam-novib/geschiedenis) (8.1.2018). 
Westschweizer leiteten die Einladung an Anne-Marie Holenstein weiter, die am Treffen teilnahm. ${ }^{129}$ Damit war der Informationsfluss vom internationalen Genf zur nationalen NGO in Zürich erfolgt. Anne-Marie Holenstein traf als Vertreterin der nationalen, säkularen Nicht-Regerungsorganisation EvB auf Vertreter ähnlicher Organisationen und erfuhr dort, wie die Niederländer bereits 1968 mit dem Rohrzucker auf einen tropischen Rohstoff als Symbol für ihre Kampagne für gerechteren Nord-Süd-Handel gesetzt hatten. ${ }^{130}$

Das Vorhaben der Niederländer, ihre Rohrzucker-Kampagne von 1968 europaweit koordiniert zu wiederholen, verlief jedoch im Sand. Die Briten preschten im Hinblick auf einen bevorstehenden Beitritt Grossbritanniens zur EWG auf eigene Faust vor. ${ }^{131}$ Im Falle der Schweiz stellte die EvB 1970 noch keine schlagkräftige Organisation dar, zudem fehlte hier im Gegensatz zu Grossbritannien und den Niederlanden die Notwendigkeit, die Zugehörigkeit oder den bevorstehenden Beitritt des eigenen Landes zur EWG kritisch zu begleiten. So fanden die Niederländer in der Schweiz keine bestehende Organisation vor, die mit ihnen wie etwa britische NGOs ein koordiniertes Vorgehen besprochen hätte. ${ }^{132}$

In der Folge der Publikation der „Haslemere Declaration“ im April 1968 entstanden in Grossbritannien mehrere lokale Aktionsgruppen, die kurz durch einen Newsletter vernetzt waren. ${ }^{133}$ Der Aufbau einer Organisation scheiterte jedoch und die sogenannte Haslemere Group verschwand Mitte der 1970er-Jahre. Doch in den Jahren nach 1968 organisierten die Haslemere Aktionsgruppen mehrere Kongresse und gaben etwa ein Dutzend Publikationen heraus. ${ }^{134}$ Im April 1969 nahmen Rudolf Strahm, Martin Meyer, der in Genf studierte und dort in der lokalen EvB-Gruppe aktiv war, sowie eine Frau namens Edith Stricker am Studentenkongress „On Race and Poverty“ in Manchester teil. Strahm schrieb darüber einen Bericht und erwähnte, Exemplare der „Haslemere Declaration“ könnten bei ihm bezogen werden. ${ }^{135}$

Ein Mitglied der Haslemere Group, der junge Ökonom Jonathan Power (`1941), schrieb sehr ähnlich wie Rudolf Strahm später ein für Laien verständli-

129 SozArch, EvB, Ar 430.10.1. Anne-Marie Holenstein an Novib, 2.1.1970.

130 A.-M. Holenstein-Hasler, R. Renschler, R. H. Strahm: Entwicklung heisst Befreiung, 50.

131 Dam, Peter Van: Moralizing Postcolonial Consumer Society.

132 Ebd.

133 M. Hilton: International Aid and Development NGOs. Bodleian Library, Oxford. Oxfam archive, MS. Oxfam. COM/3/1/12. Folder 3: Haslemere Declaration. Bodleian Library, Oxford. North London Haslemere Group: Paper Tiger, London, 1969-1970.

134 Siehe dazu die Publikationsliste in der vermutlich letzten Publikation. Social Science Library, Oxford. North London Haslemere Group: Who needs the Drug companies? London, [1976?]. 135 SozArch, EvB, Ar 430.25.1. EvB, Anfangszeit: Bulletin der Evangelischen Studentengemeinde Zürich, Juni 1969. 
ches Buch über Entwicklungsökonomie. ${ }^{136}$ In einer Broschüre zur Pulverkaffeeaktion empfahl die EvB einen Dokumentarfilm von Power mit dem Titel „Macht der Armen“ über die Themen Rohstoffhandel, Kaffee-Markt und Tansania. ${ }^{137}$ Power war mit Lukas Vischer bekannt, der ihn im Auftrag des ÖRK ein Buch über den Welthunger schreiben liess und ihm Anne-Marie Holenstein als Koautorin vermittelte. ${ }^{138}$ Unter dem Titel „World of Hunger“ kam 1976 das von Power und Holenstein gemeinsam verfasste Buch heraus. ${ }^{139}$ Die Schweizer und Engländer fokussierten zunächst intellektuell auf die „Bewusstseinsbildung“. ${ }^{140}$ Dies spiegelt sich auch in der Beobachtung von Clare Saunders wider, die im Fall der britischen Dritte-Welt-NGOs bereits von deren dritten Entwicklungsphase spricht, in der über die technischen Aspekte des Welthandels diskutiert wurde. ${ }^{141}$ Die Holländer traten jedoch bereits 1968 aktivistisch hervor, als sich die „Jugendfraktion“ in der Schweiz noch nicht einmal gebildet hatte. Nach dem Frühling 1968 starteten niederländische Dritte-Welt-Aktivisten eine Rohrzucker-Kampagne, auf deren Höhepunkt sie im Dezember des gleichen Jahres Rohrzuckerportionen an alle Mitglieder des holländischen Parlaments verteilten und einen übergrossen Weihnachtsmann mit einem Herz aus tropischem Zuckerrohr vor dem Parlamentsgebäude postierten. ${ }^{142}$ Sowohl die Briten, die kurz vor dem Beitritt zur EWG standen, wie auch die Holländer, die seit 1957 zur EWG gehörten, befassten sich mit den Auswirkungen der Europäischen Einigung auf die Dritte Welt. ${ }^{143}$ In der Schweiz setzte sich Christoph Eckenstein, der später auch verschiedene südliche Länder in ihren Verhandlungen mit der EWG beriet, intensiv mit dem Thema auseinander. In der Publikation zur interkonfessionellen Konferenz im Bundeshaus von 1970 wird denn auch die Rolle der Schweiz in der europäischen Einigung angesprochen. Die Teilnehmerinnen und Teilnehmer hielten fest, dass die Schweiz keinen Vertragswerken beitreten solle, die sich für die Dritte Welt nachteilig auswirken würden. ${ }^{144}$ In den Jahren nach 1968 diskutierten die Briten den Beitritt zum EWG, der schliesslich 1973 trotz Einwänden von Seiten der Dritte-

136 J. Power: Development Economics.

137 SozArch, EvB, Ar 430.27.1. Kaffee Ujamaa 73-77: Solidaritätskaffee „Ujamaa“, 1975, Broschüre.

138 A.-M. Holenstein-Hasler, R. Renschler, R. H. Strahm: Entwicklung heisst Befreiung, 70.

139 Power, Jonathan; Holenstein, Anne-Marie: World of Hunger. A Strategy for Survival (London 1976).

140 SozArch, EvB, Ar 430.10.2. Rudolf Strahm, Entwicklungspolitische Bewusstseinsbildung Thesen für die zukünftige Arbeit, undatiert.

141 C. Saunders: British Humanitarian, Aid and Development NGOs.

142 Dam, Peter Van: Goodbye, Dam, Peter Van: It’s Not Just About Europe.

143 Dam, Peter Van: Moralizing Postcolonial Consumer Society.

144 H. Schmocker, M. Traber: Schweiz-Dritte Welt. 
Welt-Bewegung erfolgte. Die Niederlande hingegen gehörten zu den Gründerstaaten der EWG, und der niederländische Politiker Sicco Mansholt hatte die Gemeinsame Europäische Agrarpolitik (GAP) massgeblich geprägt. ${ }^{145}$ An dieser Gemeinsamen Agrarpolitik entzündete sich die Kritik der Dritte-Welt-Aktivistinnen und -Aktivisten. Der „New Internationalist“, das Sprachrohr der britischen Dritte-Welt-Bewegung, brachte unter anderem im Juni 1973, als Grossbritannien der EWG beitrat, einen Beitrag über das Thema Zucker. Aufgezeigt wurde, wie die GAP europäische Zuckerrüben dem karibischen Rohrzucker vorziehen würde und damit sogar tropische Rohstoffe, die überhaupt den Weg in die Festung Europa fänden, substituieren würde. ${ }^{146}$ Für die Schweizer Dritte-Welt-Bewegung war der „New Internationalist“ eine wichtige Informationsquelle. ${ }^{147}$ Die Niederländer hatten indessen wie erwähnt bereits seit 1968 versucht, am Beispiel Zucker eine internationale Kampagne aufzuziehen, die das Thema ins Bewusstsein der Bevölkerung bringen und zu konkreten politischen Verbesserungen führen würde. ${ }^{148}$ In den Akten der EvB sind denn auch englischsprachige Unterlagen zur Zuckerproblematik von 1970 vorhanden. ${ }^{149}$ Anne-Marie Holenstein vernetzte sich schnell und gut mit den holländischen Aktivisten.

Bereits ein Jahr nachdem sie das Sekretariat der EvB übernommen hatte, im April 1970, besuchte Holenstein in den Niederlanden wie erwähnt den internationalen Kongress der Novib in Egmond aan Zee. ${ }^{150}$ Danach war sie gut informiert über frühe Aktionen in den Niederlanden und berichtete 1972 den Bananenfrauen in Frauenfeld darüber.

Als Vertreterin der EvB sah ich meine Rolle darin, Informationen über Erfahrungen mit Konsumentenaktionen weiterzugeben, z.B. jene der Holländer mit Zucker- und Kaffeeaktionen. ${ }^{151}$

145 Van Merriënboer, Johan: Mansholt. A Biography (Brüssel 2011). Zur Rolle Mansholts als Sündenbock der GAP siehe E. F. Schumacher: Es geht auch anders, 40 - 42. Schumacher kritisiert Sicco Mansholt, der in den 60er-Jahren noch der grösste Wachstumsapostel war.

146 Ian Haig, E.E.C. and Third World to clash on sugar, The New Internationalist, June 1973. 147 SozArch, EvB, Ar 430.25.1. EvB, Anfangszeit: Aktion und Information. Möglichkeiten für Gruppen, EvB, April 1974.

148 Dam, Peter Van: Moralizing Postcolonial Consumer Society.

149 SozArch, EvB, Ar 430.10.1. International Working Congress of Action Groups on International Development, Egmond aan Zee, 1.-4.4.1970.

150 A.-M. Holenstein-Hasler, R. Renschler, R. H. Strahm: Entwicklung heisst Befreiung, 50. 151 Ebd., 63. 
Doch sowohl die internationale Zuckerkampagne wie auch die Aktionen der Haslemere Gruppen verliefen zu Beginn der 1970er-Jahre im Sand. ${ }^{152}$ Stattdessen entstanden in den 1970er-Jahren in den Niederlanden und in Grossbritannien genau wie in der Schweiz Dritte-Welt-Läden. ${ }^{153}$ Gerade im Falle Grossbritanniens bestand bereits die Tradition der charity shops, von Freiwilligen geführte Brockenstuben, deren Erlös einem guten Zweck zukam. So führte die britische DritteWelt-Organisation Oxfam in den 1960er-Jahren zahlreiche Läden, deren Erlös der Organisation zugute kam. Entsprechend war ein fliessender und sehr früher Übergang von der Wohltätigkeit zum Verkauf von Produkten aus Entwicklungsländern möglich. ${ }^{154}$ Der Verkauf von Dritte-Welt-Produkten wurde in der Schweiz $\mathrm{zu}$ einem Bestandteil des Aktivismus, aber erst einige Zeit nachdem dies in Grossbritannien und den Niederladen der Fall war. Auch die Entstehung von Dritte-Welt-Läden erfolgte hier etwas später als in den anderen beiden Ländern, nämlich Mitte der 1970er-Jahre. Die Schweizer Bewegung blieb somit im internationalen Vergleich länger „theoretisch“ in ihrem Einsatz für gerechten Handel. Die internationale Vernetzung der EvB erfolgte jedoch über Weltläden und wird in den Akten in den frühen 1970er-Jahren deutlich. Hierbei ging es fast ausschliesslich um das Thema Alternative Trading. Der Alternative Handel war das erste Kernthema der NGO, und darum ging es auch bei ihrer Vernetzung. So fanden denn auch in den 1970er-Jahren zahlreiche internationale Zusammenkünfte von Betreiberinnen und Betreibern von Weltläden statt. ${ }^{155}$

Diese Bewegung war im Gegensatz zur Zuckerkampagne und den schweizerischen und britischen Manifesten von Dauer und führte langfristig zur Lancierung des international registrierten Fair-Trade-Gütesiegels. ${ }^{156}$ Grossbritannien, die Niederlande und die Schweiz waren federführend beim Aufbau und der Einführung des internationalen Gütesiegels. ${ }^{157}$ Es ist interessant zu sehen, dass in allen drei Ländern zunächst Aktionen vorausgingen, die unabhängig voneinander grosse Ähnlichkeit hatten. In allen drei Nationen beriefen sich die Aktivistinnen und Aktivisten auf die UNCTAD - und entweder implizit oder namentlich auf Raúl

152 Dam, Peter Van: Limits of a Success Story, M. Hilton: International Aid and Development NGOs.

153 Dam, Peter Van: Moralizing Postcolonial Consumer Society, 235.

154 M. Anderson: History of Fair Trade, 114.

155 SozArch, Claro 1010.11. SOS Niederlanden und andere Organisationen: Tagungen, Workshops zu Alternativen Handel 1973-1977.

156 M. Anderson: History of Fair Trade.

157 Seit der Einführung weisen die drei Nationen den höchsten Pro-Kopf-Konsum von zertifizierten Produkten aus. Krier, Jean-Marie: Fair Trade 2007: New Facts and Figures from an Ongoing Success Story (Culemborg 2007). 
Prebisch. In Grossbritannien kamen Mitglieder der Haslemere Group von der Universität Sussex, an der Hans W. Singer am Aufbau des Institute of Development Studies beteiligt war. ${ }^{158}$ Während Eckenstein persönlicher Berater von Prebisch gewesen war, standen Mitglieder der Haslemere Group mit dem britischen Team um Singer in Kontakt. In den Niederlanden war der Entwicklungsökonom Jan Tinbergen Teil der Bewegung. ${ }^{159}$ Alle drei nationalen Bewegungen forderten ihre Regierungen auf, die UNCTAD-Vorgaben umzusetzen und den nationalen Markt für Produkte aus Entwicklungsländern zu öffnen und vor allem im Rahmen der GAP nicht noch stärker zu schliessen. Der Marktzugang für Entwicklungsländer war jeweils das Hauptanliegen der nationalen Bewegungen. Es ist auffällig, dass sich in allen drei Ländern die ehrenamtlichen Bewegungen auflösten und kommerziell ausgerichteten Weltläden sowie professionellen NGOs Platz machten, die den Marktzugang vergassen.

Nachdem die EvB den Verkauf von Produkten 1977 in die Firma OS3 ausgelagert hatte, blieb ihr der Schwerpunkt, den Welthandel kritisch zu begleiten. ${ }^{160}$ Damit wurde sie zum Pendant der britischen Oxfam, der wohl bekanntesten Entwicklungs-NGO, die sich der Überwachung eines gerechten Welthandels verschrieben hat; unterdessen ist Oxfam weltweit tätig. ${ }^{161}$ Sie ist nach dem Zweiten Weltkrieg aus Hilfsorganisationen entstanden und hat sich in den 1960ern, als die Kriegsschäden in Europa behoben waren und Wohlstand herrschte, zur DritteWelt-NGO gewandelt. Oxfam richtete in Grossbritannien erste Dritte-Welt-Läden bzw. Alternative Trading Channels ein. ${ }^{162}$ Ähnlich wie die EvB lagerte Oxfam diese Sparte aus und trennte sie organisatorisch klar von der NGO-Arbeit. Anders als die EvB war Oxfam jedoch stets in der klassischen Entwicklungszusammenarbeit tätig und spurte in diesem Kontext den Übergang von der reinen Hilfe zur Hilfe zur Selbsthilfe mit dem Fokus auf den Kleinbauern früh vor. ${ }^{163}$

Aus Schweizer Sicht ist Oxfams Rolle in Tansania von Bedeutung. Tansania galt in der frühen Dritte-Welt-Bewegung als Musterland. ${ }^{164}$ Julius Nyerere, der erste Präsident nach der Unabhängigkeit des Landes im Jahr 1961, war auch ein bedeutender afrikanischer Intellektueller. Westliche Studenten lasen seine Schriften mit grossem Enthusiasmus. Nyereres Idee eines afrikanischen Sozialismus, genannt Ujamaa, bot den westlichen Aktivistinnen und Aktivisten eine

158 R. Jolly: A Short History.

159 Dam, Peter Van: Moralizing Postcolonial Consumer Society.

160 Siehe dazu Kapitel 4.1. Die EvB und die OS3: Bücher und Nahrungsmittel.

161 https://www.oxfam.org/ (14.9.2017).

162 M. Anderson: History of Fair Trade.

163 M. Black: Oxfam the First 50 Years, 69.

164 M. Jennings: ,Almost an Oxfam in Itself‘. 
Alternative bei der Auswahl zwischen dem Kapitalismus und dem Sowjetmarxismus des Kalten Kriegs. In neuerer Forschung wurde nun aufgezeigt, wie eng Nyerere von Jimmy Betts, einem ehemaligen britischen Kolonialbeamten, beraten wurde. Betts wechselte fliessend von der britischen Kolonialverwaltung zu Oxfam, für die er als field director in Tansania stationiert war. Bereits in den späten 1960er-Jahren nahm die Ujamaa-Politik in Tansania totalitäre Züge an, was zu Zwangsumsiedlungen von sechs Millionen Menschen in Ujamaa-Modelldörfer führte. Während die alternative Bewegung die Wachstumskritik und Small is beautiful euphorisch als neue Leitideen aufnahm, entwickelte sich die tansanische Wirtschaftspolitik genau in die gegenteilige Richtung. Immer grössere landwirtschaftliche Einheiten sollten immer mehr produzieren. So forderte die Iringa Erklärung 1972 eine Steigerung der Produktion und eine Modernisierung der Landwirtschaft. Die „villagization“ bedeutete im Kontext der Landreform in Tansania nicht eine Verkleinerung, sondern eine Vergrösserung der landwirtschaftlichen Produktionseinheiten. ${ }^{165}$

Jimmy Betts unterstützte Nyerere und dessen Politik jedoch weiterhin und benutzte Tansania als Idealtypus für das Publikum in Grossbritannien. Unter der Studentengeneration von 1968 herrschte eine eigentliche „Tanzaphilia“. Zahlreiche junge Menschen aus Europa sympathisierten mit Tansania und viele reisten selbst in das ostafrikanische Land, um dort in Ujamaa-Dörfern aktiv an der Alternative mitzuarbeiten. Auch die späteren EvB-Sekretäre Rudolf Strahm, Regula Renschler und Al Imfeld, das spätere Komitee-Mitglied der EvB, bereisten Tansania. ${ }^{166}$ Al Imfeld traf Nyerere sogar persönlich. ${ }^{167}$ Aus der Korrespondenz zwischen Jimmy Betts und dem Oxfam-Hauptquartier in Oxford wird ersichtlich, dass Oxfam in diesen jungen Leuten zukünftige langjährige Spender sah. Es lag somit nicht im Interesse von Oxfam, die illusorische Vorstellung eines afrikanischen Sozialismus, wie er in den Augen der Europäer in Tansania bestand, zu zerstören und der „Tanzaphilia“ Abhilfe zu schaffen. So hielt Oxfam, die in Tansania vor Ort einen erfahrenen field director bezahlte, relevante Kritik an Nyerere unter Verschluss und profitierte zuhause in Grossbritannien vom falschen Bild, das die tansaphilen Studierenden sich machten. ${ }^{168}$

Diese eigentlich britische Studie ist für diese Arbeit deshalb relevant, weil auch die Schweizer Studierenden von der „Tanzaphilia“ erfasst wurden. Die Berner Arbeitsgruppe Schweiz-Dritte Welt (AG3W), zu der hauptsächlich Theo-

165 Ebd., 511.

166 A.-M. Holenstein-Hasler, R. Renschler, R. H. Strahm: Entwicklung heisst Befreiung, 88 - 89, 113.

167 L. Suter: In aller Welt.

168 M. Jennings: ,Almost an Oxfam in Itself‘. 
logiestudenten, aber auch andere wie etwa Rudolf Strahm gehörten, lancierte 1973 zum ersten Mal eine Kampagne zum Verkauf von Ujamaa-Pulverkaffee aus Tansania. ${ }^{169}$ Rudolf Strahm reiste 1974, nach Abschluss seiner Arbeit bei der UNCTAD, ebenfalls nach Tansania, wo er einige Wochen lang in einem UjamaaDorf lebte. ${ }^{170}$ Nach seiner Rückkehr trat er seinen Posten als Sekretär der EvB an und lancierte in deren Namen ein zweites Mal eine Aktion für Ujamaa-Pulverkaffee. ${ }^{171}$ In diesem Rahmen entstand auch eine Broschüre zur Verkäuferschulung, in der die Ujamaa-Philosophie und Tansania modellhaft beschrieben waren. ${ }^{172}$ Damit wurde auch die EvB als Organisation Teil der „Tanzaphilia“, die den afrikanischen Sozialismus zum Ideal hochstilisierte und die Augen vor der Realität verschloss. Wie das Oxfam-Hauptquartier in Oxford erreichten auch die EvB in Zürich interne Berichte, die vor einer totalitären Entwicklung in Tansania warnten. Ein Schweizer Filmteam schrieb der EvB im September 1974 aus Tansania: „Das politische Programm von Ujamaa und Self-Reliance und die Wirklichkeit Tansanias scheinen weiter zu divergieren, als wir bisher glaubten. “173 Während sich die EvB intern mit Berichten von Schweizern vor Ort auseinandersetzen musste, wurde sie in den Medien von antikommunistischen Kreisen um das PR-Büro Farner mit Leserbriefen angegriffen. Die Leserbriefe warfen der EvB die Unterstützung eines sozialistischen Unrechtsregimes vor und behaupteten, es seien Gelder der Kampagne abgezweigt worden. Die EvB belegte die Verwendung ihrer Gelder, verteidigte aber die Regierung Nyerere. ${ }^{174}$ Im Rückblick muss festgestellt werden, dass die EvB mit Naivität - wenn nicht sogar mit aktiver Ignoranz - um die politische Situation in Tansania in die internationale NGO-Bewegung gestartet ist.

$169 \mathrm{Zu}$ einer ausführlichen Auswertung der Ujamaa-Kampagne siehe K. J. Kuhn: Fairer Handel und Kalter Krieg.

170 Fischer, Rahel; Schär, Manuel: ,Es gab auch eine andere Schweiz': Rudolf Strahm, in: Bernhard C. Schär, Ruth Ammann, Thomas Färber, Stefan Bittner, Markus Hofer, Yves Niederhäuser, Vera Sperisen, Marc Griesshammer, Rahel Fischer, Manuel Schär, Renate Schär, Evelyne Vaudan (Hg.): Bern 68. Lokalgeschichte eines globalen Aufbruchs - Ereignisse und Erinnerungen (Baden 2008) 156-162.

171 A.-M. Holenstein-Hasler, R. Renschler, R. H. Strahm: Entwicklung heisst Befreiung, 129.

172 SozArch, EvB, Ar 430.27.1. Mappe 1: Christoph Hug, Rudolf H. Strahm: Z. B. Kaffee Ujamaa, Dossier zur Verkäuferschulung, März 1975.

173 SozArch, EvB, Ar 430.27.1. Anni und Hans Sonderegger, Esther und Andreas Enderli and Freunde und Bekannte, Dar es Salaam, Tanzania, 19.9.1974.

174 K. J. Kuhn: Fairer Handel und Kalter Krieg, 30 - 38. 


\subsection{Vernetzung mit der Jugend- und Politbewegung}

Die politische Anwaltschaftlichkeit der Studierenden für die Dritte Welt und insbesondere für Afrika begann bereits vor 1968. Regula Renschler, die 1974 als Sekretärin zur EvB stiess, begann bereits 1959 als Studentin, sich mit der Dritten Welt auseinanderzusetzen. ${ }^{175}$ Wie in andere europäische Länder kamen junge Menschen aus der Dritten Welt in den 1950er- und 60er-Jahren als Austauschstudentinnen und -studenten auch in die Schweiz. Der Einfluss dieser Studierenden auf die spätere, sich nach 1968 formierende Dritte-Welt-Bewegung ist bedeutend. ${ }^{176}$ Das Institut africain in Genf, aus dem später das IUED werden würde, war ursprünglich gegründet worden, um die zahlreichen afrikanischen Austauschstudenten in Genf $\mathrm{zu}$ beherbergen und auszubilden. ${ }^{177}$ In der Deutschschweiz gehörte Regula Renschler zu den Gründerinnen und Gründern einer schweizerischen Kontaktstelle, die Studierende aus Entwicklungsländern unterstützte. ${ }^{178}$ Als das Schweizerische Institut für Auslandforschung 1964 zu einem Vortragszyklus unter dem Titel „Aktuelle Probleme Afrikas“ mehrere Verteidiger der Apartheid in Südafrika einlud, gehörte Regula Renschler zu den Verfassern eines offenen Protestbriefes. ${ }^{179}$ Sie besuchte 1965 Algerien, 1966 arbeitete sie während eines Jahres in fünf verschiedenen afrikanischen Ländern als Ausbilderin von Journalisten. Im Februar 1969 reiste Renschler mit dem Schweizer Diplomaten August R. Lindt nach Nigeria und alleine weiter nach Biafra, wo sie mehrere Monate lang blieb, bis sie und Lindt im Juni 1969 von der nigerianischen Regierung des Landes verwiesen wurden. ${ }^{180}$ Es überrascht nicht, dass Renschler 1970 als Redaktorin der „Schweizerischen Arbeiterzeitung“ auf der interkonfessionellen Konferenz Schweiz-Dritte Welt in Bern zugegen war. ${ }^{181}$ Hatte sie sich doch bereits über ein Jahrzehnt mit der Nord-Süd-Thematik befasst und DritteWelt-Länder bereist.

Rudolf Strahm begann im Mai 1968 sein Ökonomiestudium in Bern. ${ }^{182}$ Ein Jahr später, im Frühling 1969, reiste er mit Kollegen auf den Studentenkongress in

175 A.-M. Holenstein-Hasler, R. Renschler, R. H. Strahm: Entwicklung heisst Befreiung, 78.

176 Q. Slobodian: Foreign Front.

177 M. Nobs-Margairaz: L'Institut africain, 27.

178 A.-M. Holenstein-Hasler, R. Renschler, R. H. Strahm: Entwicklung heisst Befreiung, 78.

179 Q. Slobodian: The World Economy, A.-M. Holenstein-Hasler, R. Renschler, R. H. Strahm: Entwicklung heisst Befreiung, 84.

180 Perrenoud, Marc: Lindt, August R., in: Marco Jorio (Hg.): Historisches Lexikon der Schweiz (Basel 2002-2014) http://www.hls-dhs-dss.ch/textes/d/D14864.php (24.1.2008), A.-M. Holenstein-Hasler, R. Renschler, R. H. Strahm: Entwicklung heisst Befreiung, 92.

181 Ebd., 97.

182 Ebd., 116. 
Manchester, wo er sich über die Aktivitäten und Denkschulen der Aktivisten in Grossbritannien informierte. ${ }^{183}$ Strahm gründete mit Theologiestudentinnen und -studenten die Arbeitsgruppe Biafra, aus der 1969 die AG3W hervorging. ${ }^{184}$ Diese Arbeitsgruppe sollte denn auch die Idee einbringen, ein Produkt als Symbol für den gerechten Nord-Süd-Handel zu lancieren. ${ }^{185}$

Weder Renschler noch Strahm vertraten radikal marxistische Positionen. ${ }^{186}$ Sie stehen wohl repräsentativ für die schweizerische Jugendbewegung von 1968, die zurückhaltender agierte als jene in anderen europäischen Städten. ${ }^{187}$ Renschler und Strahm repräsentieren auch die Kontinuität, die vor der Schablone des Jahres 1968 als radikales Umbruchsmoment erst recht zu Tage tritt. Bereits in den Jahren vor 1968 hatte Renschler sich für Studierende aus der Dritten Welt eingesetzt und zur sozialdemokratischen Schweizer Geschichte geforscht. ${ }^{188}$ Ebenfalls vor 1968, im Rahmen des Protests gegen den Vortragszyklus des Instituts für Auslandforschung, erhielten die Studierenden Rückendeckung von der „Neuen Zürcher Zeitung“ (NZZ), die wie kein anderes Blatt das Schweizer Establishment verkörperte. ${ }^{189}$ Im Oktober 1967, sozusagen als Auftakt des Jahres 1968, wurde der junge Anwalt Jean Ziegler für die Genfer Sozialisten in den Nationalrat gewählt. ${ }^{190}$ Erstaunlich, dass der Name Jean Ziegler in den Unterlagen der EvB kaum vorkommt. Auch mit dem Komitee in der Romandie scheint Ziegler nicht näher in Kontakt gewesen zu sein. Dies ist umso erstaunlicher, als er eine Parlamentariergruppe Schweiz-Dritte Welt präsidierte. ${ }^{191}$ Strahm hörte an der Universität Bern bei Jean Ziegler Vorlesungen zur Entwicklungspolitik, doch eine konkrete gemeinsame ausserparlamentarische Arbeit von Ziegler und der Bewegung um die „Erklärung von Bern“ ist nicht nachweisbar. ${ }^{192}$ Im Falle von Zieglers Postulat für eine Schaffung eines „schweizerischen Instituts für die Probleme der Dritten

183 Siehe Kapitel 2.4. Die internationale Vernetzung der Erklärung von Bern.

184 A.-M. Holenstein-Hasler, R. Renschler, R. H. Strahm: Entwicklung heisst Befreiung, 117.

185 Ebd., 44-45.

186 SozArch, EvB, Ar 430.62.8 IDA. R. Strahm an Pfr. E. J., 11.6.1976.

187 B. C. Schär: ,1968‘ als wiederbelebte bürgerliche Revolution, 7.

188 A.-M. Holenstein-Hasler, R. Renschler, R. H. Strahm: Entwicklung heisst Befreiung, 78.

189 Q. Slobodian: The World Economy, A.-M. Holenstein-Hasler, R. Renschler, R. H. Strahm: Entwicklung heisst Befreiung, 84, Silberschmidt, Max: Das Schweizerische Institut für Auslandforschung, 1943-1981 (Zürich 1981) 28-30.

190 J. Wegelin: Jean Ziegler.

191 Le Comte, Guy: Ziegler, Jean, in: Marco Jorio (Hg.): Historisches Lexikon der Schweiz (Basel 2002-2014) http://www.hls-dhs-dss.ch/textes/d/D33772.php (26.2.2014).

192 A.-M. Holenstein-Hasler, R. Renschler, R. H. Strahm: Entwicklung heisst Befreiung, 118. 
Welt“ war es wiederum so, dass die Aussichtslosigkeit des Postulats auf Zieglers Person zurückzuführen war. ${ }^{193}$

Die jungen Aktivisten positionierten sich selbst zwar nicht radikal links, vertraten aber eine Haltung, die leicht von der UNCTAD-Doktrin, auf die in der „Erklärung von Bern“ verwiesen war, abwich. Und tatsächlich bedeutete diese leichte Abweichung, dass die Jugendfraktion die Grundzüge der marxistisch-leninistischen Imperialismustheorie gemäss Galtung, Stavenhagen, Senghaas oder letztlich Jean Ziegler vertraten, die in den privaten westlichen Grosskonzernen eine Verlängerung des westlichen Imperialismus mit anderen Mitteln sah. ${ }^{194}$ Diese Multi-Kritik, der „anticorporatism“, wurde bereits auf der Konferenz von 1970 laut und fiel beim älteren theologischen Establishment durchaus auf fruchtbaren Boden. ${ }^{195}$ Im Vorfeld der Konferenz hatten sich Mitglieder des Komitees der EvB mit Vertretern der „Jugendfraktion“ getroffen und „das Vorgehen“ besprochen. ${ }^{196}$ Auch wenn die älteren Theologen zunächst mehr Engagement der privaten Industrie in Entwicklungsländern gefordert hatten, so war kaum eine Verteidigung der westlichen Grosskonzerne von ihnen zu erwarten. Die christliche Bescheidenheit deckte sich mit der neokonservativen, antimodernistischen Kritik an der „Vermassung“ der bürgerlichen Schweiz. ${ }^{197}$

Auf der Konferenz Schweiz-Dritte Welt, die im November 1970 im Bundeshaus stattfand, zeichnete sich gemäss Kalt ein Mentalitätswechsel ab: Die Wortführerschaft ging von der Generation der Barthianer auf die Aktivisten der 68erGeneration über. ${ }^{198}$ Hier fand die Rezeption der UNCTAD-Anliegen in der Schweiz ihren Höhepunkt, gleichzeitig wurde aber der Abschied von der Urform der Doktrin eingeleitet. Zum einen gehörte Christoph Eckenstein zu den wichtigen Figuren der Konferenz, der als persönlicher Mitarbeiter Prebischs die Anliegen der UNCTAD wie seine Hostentasche kannte. Eckenstein und Prebisch, der auf der ersten Session sprach, erhielten von allen Seiten Zustimmung, auch wenn vermutlich viele Anwesende nur bedingt die ökonomischen Grundlagen nachvollziehen konnten. ${ }^{199}$ Jemand der dazu imstande war, war Ökonomiestudent Rudolf Strahm. Die AG3W, zu der Strahm gehörte, war ebenfalls auf die Konferenz im

193 M. Nobs-Margairaz: L'Institut africain, 65.

194 M. Schär: Strukturveränderungen statt Entwicklungshilfe, 33-38.

195 N. Klein: No Logo, xviii.

196 SozArch, EvB, Ar 430.10.1. Schweizer Komitee 1970-1973 Anne-Marie Holenstein an Mitglieder des Komitees „Erklärung von Bern“, Zürich, 10.11.1970.

197 Röpke, Wilhelm: Die Gesellschaftskrisis der Gegenwart (Erlenbach-Zürich 1942), 316.

198 M. Kalt: Tiersmondismus in der Schweiz, 296-303.

199 H. Schmocker, M. Traber: Schweiz-Dritte Welt, 24. 
Bundeshaus geladen und hatte ihre eigenen Anliegen formuliert. ${ }^{200}$ Die „Jugendfraktion“ prägte einen Slogan, der die EvB für die nächsten Jahrzehnte leiten sollte und zugleich auch die Abkehr von den ursprünglichen UNCTAD-Anliegen markierte. Die AG3W schrieb:

Wir sind das Problem. Entwicklungshilfe im üblichen Sinn führt nicht zum Ziel; nötig sind Strukturveränderungen in den internationalen Wirtschaftsbeziehungen; dies setzt Strukturveränderungen in unserem Lande voraus. In der Entwicklungspolitik kommt es nicht so sehr darauf an, mehr zu geben, als vielmehr weniger zu nehmen. ${ }^{201}$

Der neue Slogan, der das zukünftige paraakademische Fair-Trade-Konzept auf den Punkt bringen sollte, hiess nun „Weniger nehmen“. Er ersetzte den Slogan Trade, not Aid. Oder genauer: Not Aid wurde mit dem ersten Halbsatz „es kommt nicht so sehr darauf an, mehr zu geben“ beibehalten, doch Trade änderte sich in „Weniger nehmen“.

Aus akademisch-ökonomischer Sicht liesse sich der Slogan „Weniger nehmen“ in ein Plädoyer für nationale Autarkie und ein Schrumpfen des Welthandels übersetzen. Sehr klar zeigt diese widersprüchliche Äusserung der Gruppe auf, dass sie einerseits die Öffnung des Schweizer Marktes für Industrieprodukte aus der Dritten Welt verlangte, während sie andererseits unfreiwillig auch den Slogan aufsetzte, der den Neoprotektionismus der kommenden Jahre legitimieren würde. Ursprünglich bezog sich das „Weniger nehmen“ im Kontext von 1970 insbesondere auf die Kapitalflucht und nicht auf den Import von Agrarprodukten. Denn auf der Konferenz von 1970 herrschte noch das Bild der Entwicklungsländer der 1960er-Jahre vor, das Aufbruchsstimmung, neuen westlichen Wohlstand und eine selbstbewusste, zum Teil aber korrupte Elite zeigte. 1970 galten die Entwicklungsländer noch als ernstzunehmende, politisch und finanziell souveräne Staaten, denen eine bedeutende Zukunft und ein starkes Wirtschaftswachstum bevorstand. Die „Euphorie“ herrschte noch ungebrochen vor. ${ }^{202}$ Dies erklärt, dass sich die Bewegung in den 1970er-Jahren kaum um den zunehmenden Agrarprotektionismus kümmerte. Sie forderte, der Finanzplatz Schweiz sowie die Fluchtgelder von Diktatoren aus dem Süden müssten unter die Lupe genommen werden. So sollte sich vor allem Rudolf Strahm dieses Themas annehmen und sozusagen

200 M. Kalt: Tiersmondismus in der Schweiz, 296-303.

201 A.-M. Holenstein-Hasler, R. Renschler, R. H. Strahm: Entwicklung heisst Befreiung, 37-38. 202 R. Quaas: Fair Trade, 146. 
eine Spin-off-NGO der EvB gründen: die Aktion Finanzplatz Schweiz. ${ }^{203}$ Strahm verliess denn auch 1978 die EvB, um für die Sozialdemokratische Partei (SP) die sogenannte Bankeninitiative aufzuziehen. ${ }^{204}$

Während Anne-Marie Holenstein zu Beginn der 1970er-Jahre das Sekretariat der EvB führte, meldeten sich aus dem Umfeld der AG3W Leute, die Kampagnen durchführen wollten. ${ }^{205}$ Der Übergang von der intellektuellen Forderung an die Politik - in Form einer schriftlichen Erklärung - zur Kampagne markierte auch die Übernahme des Zepters durch die jüngere Generation. Damit fand ein Mentalitätswechsel statt. Die ältere Generation hatte sich noch an der christlichen Introspektion orientiert und das Veränderungspotential bei sich selbst gesehen, im eigenen Land, sowie das Urprinzip des Almosens vertreten. Auch die jungen Studierenden der Theologie sagten noch: „Wir sind das Problem““. ${ }^{206}$ Faktisch in Kampagnen übersetzt meinten die Studierenden mit dem „wir“ jedoch nicht sich selbst, sondern die bürgerlich-konservative Vätergeneration, sprich den Staat und die Privatwirtschaft. Sie prangerten die multinationalen Konzerne sowie die Schweizer Regierung an und legten damit auch implizit eine Selbstgerechtigkeit an den Tag, die neu war. Diese Selbstgerechtigkeit brachte sie in Konflikt mit der christlichen, selbstkritischen Haltung der Vätergeneration und entwickelte sich in ihrer ganzen Widersprüchlichkeit zu einem Grundstein der EvB. ${ }^{207}$ Die EvB fusionierte die Selbstkritik der christlichen ersten Generation mit der Selbstgerechtigkeit der aktivistischen zweiten Generation, indem sie auf der Grundlage des Manifests Kampagnen aufbaute. Damit schuf sie sich eine moralische Autorität, von der sie lange zehren sollte. ${ }^{208}$ Mit dem Schritt zur Selbstgerechtigkeit erfolgte auch gleichzeitig der Schritt zur konkreten Kampagne und dem angewandten alternativen Handel. Weiterhin bestand das Ziel darin, in der Schweiz das Bewusstsein der Bevölkerung zu schärfen, damit eine gerechtere Handelspolitik gegenüber der Dritten Welt möglich und unterstützt würde. Mit einem

2032012 löste sich die Aktion Finanzplatz Schweiz wieder auf, die Aktivitäten werden von der EvB (neu Public Eye) fortgeführt. Das Archiv wurde dem Schweizerischen Sozialarchiv übergeben, siehe http://findmittel.ch/archive/archNeu/Ar518.html. (7.4.2020)

204 A.-M. Holenstein-Hasler, R. Renschler, R. H. Strahm: Entwicklung heisst Befreiung, 166. Siehe dazu auf der Webseite der Schweizerischen Bundeskanzlei unter der Rubrik „Volksinitiativen“: Eidgenössische Volksinitiative „gegen den Missbrauch des Bankgeheimnisses und der Bankenmacht“, abgelehnt am 20.5.1984 (https://www.admin.ch/ch/d//pore/vi/vis133.html) (14.9.2017).

205 Ebd., 44-45.

206 Ebd., 37-38.

207 M. Kalt: Tiersmondismus in der Schweiz, 296-303.

208 Körber, Achim: The Political Economy of Environmental Protectionism (Cheltenham, UK, Northampton, MA 2000). 
Hungerstreik während der interkonfessionellen Konferenz Schweiz-Dritte Welt 1970 protestierten die Vertreter der Jugendfraktion gegen die Schweizer Zollschranken gegenüber Produkten aus Entwicklungsländern. ${ }^{209}$

In den 1970er-Jahren organisierte die „Jugendfraktion“ Kampagnen und insbesondere Rudolf Strahm schrieb eigene Bücher und gab Bücher heraus. Deren Verkauf sollte der EvB in den ersten Jahren durchaus kommerzielle Einnahmen bringen. ${ }^{210}$ Langsam koppelte sich die NGO von der Universität und der Wissenschaft ab und schuf ihre eigene, paraakademische Doktrin. Die Schaffung dieser alternativen Doktrin verlief parallel zur Einführung eines alternativen Handels. In beiden Fällen war es ein sehr kleiner Wirkungsbereich, doch langfristig gewann sowohl diese „Nichtregierungslehre“ ausserhalb der universitären Wissenschaft als auch der alternative Handel ausserhalb des Handels in der Schweiz wie in Grossbritannien und in den Niederlanden an Bedeutung.

\subsection{Mit der UNCTAD-Doktrin gegen Nestlé: Ujamaa-Pulverkaffee}

Das grundsätzliche Bekenntnis der ersten Generation der EvB zu den UNCTADForderungen von 1964 ist nicht zu bestreiten. So hielt etwa Peter Gessler, nebst den Barthianern einer der aktivsten Initianten der Erklärung, 1970 fest:

Hilfe zur Selbsthilfe bedeutet, dass Entwicklungsländer eigene Industrien aufbauen, auf dem Weltmarkt als Konkurrenten auftreten und die eigene Kapitalbildung steigern können. Die Zollpolitik der Industrieländer soll die Entwicklungsländer nicht länger daran hindern, Exportindustrien aufzubauen, die für eine gedeihliche Wirtschaftsentwicklung und die Beteiligung am Welthandel unerlässlich sind. Die internationale Arbeitsteilung muss neu strukturiert werden. Die Schweiz muss zugunsten der Importe aus Entwicklungsländern Schutzzölle auf verarbeiteten Rohstoffen abbauen. ${ }^{211}$

Damit formulierte Gessler die intellektuelle Grundlage, auf welcher die „Jugendfraktion“, wie das erste Komitee der EvB die jüngere Generation um Rudolf Strahm und Al Imfeld nannte, einen ersten Produkteverkauf lancieren würde. Die erste Fair-Trade-Kampagne wurde nicht von der EvB, sondern von der AG3W lanciert. 1973 erhielt die Arbeitsgruppe eine Lieferung Ujamaa-Pulverkaffee und bereitete diese in den Räumen des Theologischen Seminars der Universität Bern zum

209 M. Schär: Strukturveränderungen statt Entwicklungshilfe, 82.

210 SozArch, EvB, Ar 430.10.2. Schweizer Komitee 1974-77: Rechnung 1974.

211 SozArch, EvB, Ar 430.25.1. EvB, Anfangszeit: Die Beziehungen der Schweiz zu den Entwicklungsländern, Entwurf M. Geiger (Punkt 5 Dr. Peter Gessler), undatiert. 
Verkauf vor. ${ }^{212}$ Fast wortgleich wie Gesslers Formulierung steht auf der Etikette, die 1973 auf die Gläser mit Pulverkaffee geklebt wurde: „Die Entwicklungsländer sollten den Kaffee selber verarbeiten können. Die dazu nötige Industrialisierung aber wird durch ihren Kapitalmangel und durch unser Zollsystem behindert. “213

Nach der ersten Kaffee-Aktion wurde Rudolf Strahm 1974 bei der EvB angestellt, im gleichen Jahr fand im evangelischen Tagungszentrum Gwatt eine weitere Tagung statt. Dort brachte Strahm die Idee vor, dass die EvB in einer Kampagne wiederum einen Ujamaa-Verkauf lancieren sollte. ${ }^{214}$ Die Kampagne wurde ausführlich diskutiert und beschlossen. So fanden denn 1973 (noch von der AG3W organisiert), 1974 und 1975 in der ganzen Schweiz Ujamaa-Pulverkaffee-Aktionen statt. ${ }^{215} 1976$ erfolgte schliesslich der Schritt zur Auslagerung des Produkteverkaufs in eine eigene Gesellschaft und 1977 die Gründung der OS3.216 Im Hinblick auf die Entwicklung des späteren Fair-Trade-Konzepts ist besonders eine Etikette interessant, die während der ersten Aktion 1973 auf die Gläser mit Pulverkaffee geklebt wurde. Darauf stand: „Gerechtigkeit muss nicht mehr kosten!““217 Auch auf den Plakaten, welche für die Verkaufsstände aufgehängt wurden, stand: „Kaffee UJAMAA ist billiger als gewöhnlicher Pulverkaffee; und doch verdient Tansania doppelt soviel wie beim Export von Kaffeebohnen, weil es diese selber verarbeitet hat.“218 Dies war eine Umschreibung des UNCTAD-Slogans Trade, not Aid und eine Paraphrasierung des fünften Punktes der „Erklärung von Bern“, wonach die ökonomischen Strukturen unseres Landes verändert werden sollten. Andererseits war es aber auch noch ein Hinweis darauf, wie stark 1973 in der Aktion der AG3W die ursprünglichen UNCTAD-Anliegen zum Zug kamen, die auf gerechte Handelsstrukturen anstatt auf Almosen in Form von freiwillig bezahlten höheren Preisen abzielten. Der Zweck der ersten Pulverkaffee-Aktion bestand darin, die Wirtschaftswissenschaften geschickt als Argument zu nutzen. Die Aktivistinnen und Aktivisten benutzen das marktwirtschaftliche Argument der freien Preisbildung als Vorteil für die Konsumentinnen und Konsumenten. Dieser marktwirtschaftliche Grundgedanke ging auf dem weiteren konzeptuellen Weg verloren und sollte sich in den 1980er-Jahren in die gegenteilige Argumentation

212 A.-M. Holenstein-Hasler, R. Renschler, R. H. Strahm: Entwicklung heisst Befreiung, 128.

213 SozArch, EvB, Ar 430.27.1. Kaffee Ujamaa 73-77: Etikette, 1973 (1).

214 A.-M. Holenstein-Hasler, R. Renschler, R. H. Strahm: Entwicklung heisst Befreiung, 124-125. 215 SozArch, EvB, Ar 430.27.1. Kaffee Ujamaa 73-77. K. J. Kuhn: Fairer Handel und Kalter Krieg, 23.

216 Siehe dazu Kapitel 3.8. Entwicklungshilfe im Inland und die Gründung der Importgenossenschaft OS3 1977.

217 SozArch, EvB, Ar 430.27.1. Kaffee Ujamaa 73-77: Etikette, 1973 (2).

218 SozArch, EvB, Ar 430.27.1. Kaffee Ujamaa 73-77: Plakat. 


\section{Warum Kaffee her- gestellt in Tansania?}

Wenn Sie Pulverkaffee kaufen, der in der Schweiz verarbeitet worden ist, bezahlen Sie durchschnittlich Fr. 50.für 1 Kilogramm. Davon gehen aber nur Fr. 10. - ins Ursprungsland. Dabei ist der Kaffeebaum schwierig zu pflegen, beansprucht den Boden stark und bringt während der ersten fünf Jahre praktisch keine Frucht.

Um $1 \mathrm{~kg}$ Pulverkaffee zu erhalten, muss der Kaffeebauer 12000 Kaffeebohnen (d. h. die Ernte von 6 Kaffeebäumen) pflücken, waschen, sortieren und schälen - das alles für 10 Franken. Das Einkommen der Kaffeebauern ist dementsprechend gering.

Die Entwicklungsländer sollten den Kaffee selber verarbeiten können. Die dazu nötige Industrialisierung aber wird durch ihren Kapitalmangel und durch unser Zollsystem behindert.

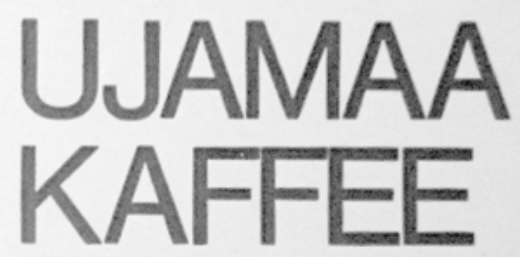

Der Kaffee mit dem Aroma der Selbstbefreiung

Reiner Kaffeeextrakt Hergestellt in TANSANIA 100 gr. ergeben ca. 66 Tassen

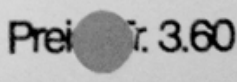

Der UJAMAA-Kaffee ist nun in Tansania selber verarbeitet worden, d. h. im Land, wo die Bohnen gepflückt worden sind. Das bedeutet:

Tansania bekommt nicht Fr. 10.-, sondern Fr. 16.- pro kg Pulverkaffee.

An der Verarbeitung gewinnen tansanische Arbeiter und nicht irgend ein Grosskonzern.

Es ergeben sich damit neue Arbeitsplätze für Tansanier.

Tansania erzielt stabile Preise für sein Produkt, denn Pulverkaffee kann gelagert werden, Kaffeebohnen jedoch nicht; sie müssen jeweils fort abgestossen werden, oft de dann, wenn der Preis extrem niedrig ist.

\section{Warum Kaffee UJAMAA?}

Dieser Kaffee ist nicht das bittere Produkt brasilianischer Hungerlöhne und Foltern.

Er hat nicht den bitteren Beigeschmack vom Blut jener Angolesen, die unter portugiesischem Terror schuften müssen.

Und er wurde nicht durch unsere Industrie verteuert.

Er kommt nämlich aus Tansania, wo er geschält, getrocknet, gebrannt und gemahlen wurde. Tansania ist jenes afrikanische Land, wo die Armen angefangen haben, sich selber zu befreien. Und zwar aus eigener Kraft.

Das Mittel zu dieser Selbstbefreiung heisst UJAMAA:

- gemeinsam planen

- gemeinsam arbeiten

- gemeinsam verdienen

- gemeinsam leben

Abb. 3: Etikette für Ujamaa-Pulverkaffee (1973) 
verkehren, denn dann wurde für den Rohkaffee ein freiwilliger Aufpreis verlangt, während der Hinweis, dass Tansania am Pulverkaffee Ujamaa doppelt so viel verdiene wie durch den Export von Kaffeebohnen von der Etikette verschwand. ${ }^{219}$ Die ursprüngliche, auf den UNCTAD-Forderungen basierende Grundidee der Bewegung ist also klar auf der Etikette beschrieben: Das Ziel bestand darin, einem Entwicklungsland zu Wirtschaftswachstum und Einkommen zu verhelfen, indem man das verarbeitete Produkt statt das Rohprodukt kaufte. Damit wurden ein freiwilliger Aufpreis, Hilfe oder Almosen überflüssig. Im Gegenteil, die Konsumentin bzw. der Konsument profitierte sogar noch im besten ökonomischen Sinne von tieferen Preisen.

In den 1970er-Jahren erfolgte einerseits eine zunehmende Professionalisierung der EvB, andererseits war aber die Zeit, in der es für Mitglieder einer Kirchgemeinde oder für Studierende völlig selbstverständlich war, Freiwilligenarbeit $\mathrm{zu}$ leisten, noch nicht vorbei. So konnte die erste Ujamaa-PulverkaffeeKampagne der EvB 1974 sowohl mithilfe eines professionellen Sekretariats als auch mit zahlreichen Freiwilligen durchgeführt werden. ${ }^{220}$ Letztere durchliefen eine Schulung, die sich - ab 1975 - inhaltlich auf die Broschüre „Z. B. Kaffee Ujamaa“ stützte. ${ }^{221}$ Strahm hatte die Schrift, die für drei Franken auch verkauft wurde, zusammen mit Christoph Hug verfasst. Strahm und Hug zeigten darin vereinfacht am Beispiel des Ujamaa-Pulverkaffees und für Laien verständlich das Problem der Verschlechterung der terms of trade bzw. der Prebisch-Singer-These (siehe Abbildung 4). Die akademische Ökonomie wurde also zunächst in einer Broschüre und in einem Schulungsseminar zusammengefasst und mit Nyereres afrikanischem Sozialismus angereichert. Die „Bewusstseinsbildung“, die „Meinungsumbildung“, stand im Vordergrund, dies war die Hauptaufgabe der freiwilligen Pulverkaffee-Verkäufer. Dieses Ziel sollte bei ihrer Gründung auch von der Firma OS3 übernommen werden:

Die Wiederverkäufergruppen und -Organisationen müssen angehalten werden, den Verkauf mit der Verbreitung von Informationen zu verbinden und regelmässige Verkäuferschulung durchzuführen. 222

219 A.-M. Holenstein-Hasler, R. Renschler, R. H. Strahm: Entwicklung heisst Befreiung, 129.

220 A.-M. Holenstein-Hasler, R. Renschler, R. H. Strahm: Entwicklung heisst Befreiung, 128.

221 SozArch, EvB, Ar 430.27.1. Mappe 1: Christoph Hug, Rudolf H. Strahm: Z. B. Kaffee Ujamaa. Dossier zur Verkäuferschulung, März 1975.

222 SozArch, Claro 1011.1. OS3 Gründung: Grundsätze der Geschäftspolitik, Juni 1977. 


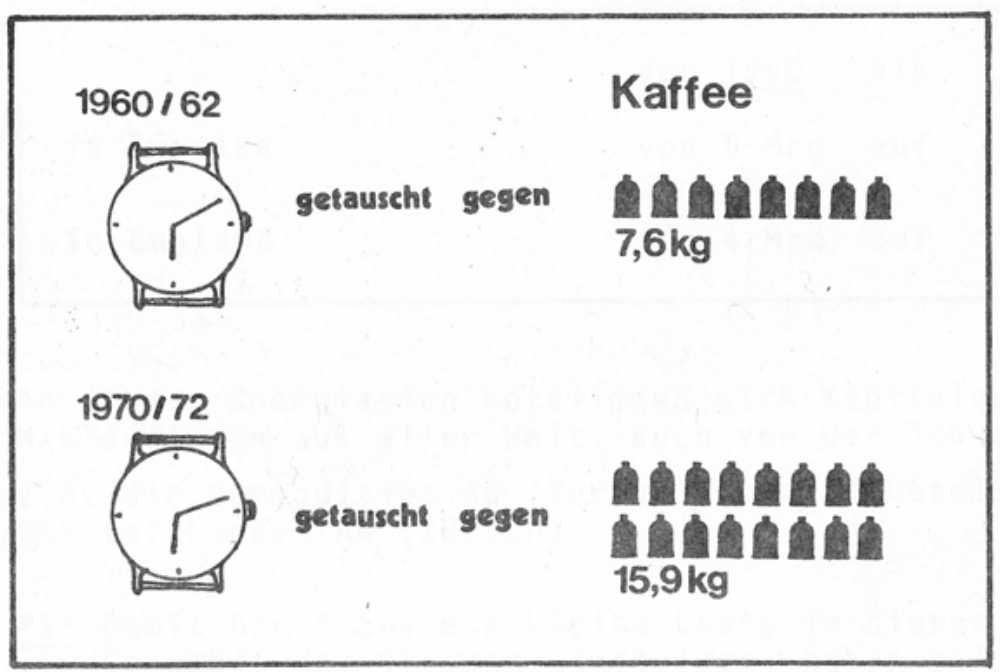

Abb. 4: Infografik der Prebisch-Singer-These (1975)

Dieser Grundsatz sollte bereits Ende der 1970er-Jahre hinfällig werden, als die OS3 erwog, doch an kommerzielle Weiterverkäufer zu liefern. ${ }^{223} 1980$ lancierte AnneMarie Holenstein gar die Reduktion der „Bewusstseinsbildung“ auf ein schriftloses Symbol auf einem Produkt, das die Konsumentin bzw. der Konsument ohne Gespräch mit einem Verkäufer im Supermarktregal finden konnte. ${ }^{224}$

Mit der Pulverkaffee-Aktion fand ein einschneidender konzeptueller Schritt statt: der Übergang vom aktiven Bürger zum passiven Konsumenten, von der Politik in die Privatwirtschaft. ${ }^{225}$ Die Frage des Nord-Süd-Handels verlor nicht nur zunehmend ihren akademischen Kontext, sondern verlagerte sich symbolisch vom Kopf in den Bauch. Anderson spricht in Bezug auf die britische Bewegung von „a shift from trade policy to shoppping choice“. ${ }^{226}$ Dieser Übergang vom Bürger zum Konsumenten konnte so fliessend und unbemerkt stattfinden, weil er

223 SozArch, Claro 1010.12. OS3 Gründung: Mario Carera, OS3 bald im breiteren Handel?, November 1979.

224 Siehe dazu Kapitel 4.5 Für Protektionismus und Autarkie, gegen Freihandel und Grosskonzerne: das Fair-Trade-Konzept zu Beginn der 1980er-Jahre.

225 Das heute bekannte Fair-Trade-Gütesiegel ist denn auch eine private, kommerziell registrierte und urheberrechtlich geschützte Marke. Siehe dazu etwa https://www.fairtrade.org.uk/ What-is-Fairtrade/Using-the-FAIRTRADE-Mark (30.1.2018).

226 M. Anderson: History of Fair Trade, 114. 
ebenfalls von der Vätergeneration frühzeitig angedacht worden war. Dies allerdings in einer sehr viel differenzierteren Form. Der Konsument kommt bei Biéler in seinen ersten Thesen vor, allerdings nur in Kombination mit dem politischen Bürger. Der Konsument ist nur ein Teil des Nord-Süd-Beziehungsnetzes, dessen sich der Europäer bewusst werden muss:

Von diesem Moment an werden die politischen Handlungen und die wirtschaftlichen Entscheidungen des einzelnen, seine ideologische Ausrichtung, sein staatsbürgerliches Engagement oder seine politische Abstinenz, seine Wahl beim Einkaufen, seine Entschlüsse, zu sparen, auszugeben oder das Geld anzulegen, seine Arbeit, seine Freizeit - zu Akten höherer Ordnung seiner Ethik, ohne dass er es bemerkt und sich dessen bewusst wird - zu Entscheidungen, die sein eigenes Los wie dasjenige seiner ihm nahe und fern stehenden Mitmenschen auf eine Weise beeinflusst, die nicht mehr rückgängig zu machen ist. ${ }^{227}$

Biéler hat damit bereits in seinem Büchlein von 1966, in dem er die „Erklärung von Bern“ vorspurte, die Idee der Konsumentenethik eingebracht. Die Referenz der Vätergeneration war jedoch weiterhin die Bibel. Diese automatische intellektuelle, moralische und emotionale Quelle verhinderte, dass die Vätergeneration der „Erklärung von Bern“ schnell und leichtsinnig aktuellen Trends folgte. Dies war nun, bei der Jugendbewegung, anders. Obwohl beispielsweise gerade bei der AG3W viele Mitglieder Theologie studierten und sich noch aktiv auf die Bibel beriefen, waren sie doch offen für neue, säkulare Trends. Ein erster solcher Trend war die „Tanzaphilia“.228

Der afrikanische Ujamaa-Pulverkaffee war für die westlichen Dritte-Welt-Organisationen das perfekte Produkt, mit dem die Prebisch-Singer-These der Konsumentin oder dem Konsumenten nähergebracht werden konnte. Der UjamaaKaffee war aber im gleichen Zug auch das perfekte „anticorporatistische“ Produkt, denn er bot den Konsumentinnen und Konsumenten eine Alternative zu Nescafé von Nestlé. Doch vor allen Dingen war der Ujamaa-Pulverkaffee das erste und letzte industriell hergestellte Produkt aus Afrika, das je von der Dritte-WeltBewegung verkauft wurde.

Tatsächlich überlebten viele der staatlichen und mit massiver staatlicher Unterstützung entstandenen infant-Industrien in Afrika die 1970er-Jahre nicht. ${ }^{229}$ Doch gerade die tansanische Pulverkaffee-Industrie bildete hier eine Ausnahme, existiert doch die Tanganyika Instant Coffee Corporation in Bukoba, aus welcher

227 A. Biéler: Gottes Gebot, 8-9.

228 M. Jennings: ,Almost an Oxfam in Itself'.

229 G. Austin: The Developmental State, 54, J. N. Bhagwati: Protectionism, 12-13. 
der Ujamaa-Kaffee stammte, heute noch. ${ }^{230}$ Doch strebte die Dritte-Welt-Bewegung bereits zur Zeit des Verkaufs von Ujamaa-Pulverkaffee nicht mehr eine richtiggehende Industrialisierung der Entwicklungsländer an, weder nach westlichem noch nach sowjetischem Modell. Fast entschuldigend wiesen Hug und Strahm bereits 1975 in ihrem Dossier zur Verkäuferschulung darauf hin, dass die Pulverkaffeefabrik in Bukoba halt aus einer Zeit stamme, „als sich Tansania noch sehr der Industrialisierung verschrieben hatte“. ${ }^{231}$ Ein Jahr vorher war die industrielle Produktion des Ujamaa-Kaffees im Ursprungsland noch als positiver Hauptpunkt der Verkaufsaktion hervorgehoben worden. So hiess es in den Unterlagen für die Verkaufsgruppen in der französischen Schweiz:

L'action Café doit permettre de transmettre le message suivant: [...] Nous ne devons pas importer seulement des matières premières des pays sous-développés (dégradation des prix) mais aussi des produits manufacturés (ex. La Tanzanie gagne $60 \%$ de plus en vendant du café soluble que si elle exportait du café en grains). ${ }^{232}$

Die Titel zweier Bücher aus dieser Zeit, die stark rezipiert wurden, stehen exemplarisch für die neue Ausrichtung des Konzepts einer gerechten Weltwirtschaft. 1972 war das Buch „The Limits to Growth“ erschienen, das vor den ökologischen Folgen eines ungezügelten industriellen Wachstums warnte. 1973 erschien „Small is Beautiful“, das herkömmliche Produktionsmethoden aufwertete und damit eine Industrialisierung der Dritten Welt nach westlichem Modell als falsch qualifizierte. ${ }^{233}$ Die beiden Titel markierten die Abkehr der westlichen Dritte-WeltBewegung von der in den 1950er- und 60er-Jahren vorherrschenden Modernisierungseuphorie. ${ }^{234}$ Die 1970er-Jahre waren somit geprägt vom Interesse der Bewegung für neue Wirtschaftsformen, wie sie im Slogan Small is beautiful verherrlicht und von der Ujamaa-Bewegung in Tansania scheinbar vorgelebt wurden. „The Age of Alternatives“: So nannte die frühere Oxfam-Aktivistin Maggie Black wie bereits erwähnt die 1970er-Jahre. ${ }^{235}$ Im englischen Sprachraum hiessen die

230 SozArch, EvB,Ar 430.27.1. Mappe 1: Christoph Hug, Rudolf H. Strahm: Z. B. Kaffee Ujamaa. Dossier zur Verkäuferschulung, März 1975. Siehe dazu die Webseite der Firma (http://www.tanica cafe.com/index.php?option=com_content\&view=featured\&Itemid=11) (28.9.2017).

231 SozArch, EvB, Ar 430.27.1. Mappe 1, Christoph Hug, Rudolf H. Strahm: Z. B. Kaffee Ujamaa. Dossier zur Verkäuferschulung, März 1975.

232 SozArch, EvB, Ar 430.10.2. Présentation du projet action café Ujamaa, September 1974.

233 E. F. Schumacher: Small is Beautiful, D. H. Meadows, D. L. Meadows, J. Randers, W. W. Behrens: Limits to Growth.

234 Siehe dazu J. Toye, R. Toye: UN and Global Political Economy, 163 und M. Schär: Strukturveränderungen statt Entwicklungshilfe, 22.

235 M. Black: Oxfam the First 50 Years, 132. 
ersten Dritte-Welt-Läden denn auch Alternative Trading Shops. ${ }^{236}$ Es scheint, dass die kurz auf die Studentenbewegung folgende Umweltbewegung (als deren Vorboten die Slogans Limits to Growth und Small is beautiful ihre Wirkung entfalteten) einen massiven Einfluss auf die Dritte-Welt-Bewegung hatte und - anders als das Jahr 1968 - einen grundlegenden Richtungswechsel bewirkte.

236 Anderson, Matthew: Fair Trade: NGOs and Fair Trade: the Social Movement behind the Label, in: N. J. Crowson, Matthew Hilton, James McKay (Hg.): NGOs in Contemporary Britain. NonState Actors in Society and Politics since 1945 (Basingstoke 2009), 222-241. 